\title{
Zytologische Untersuchungen der Hyalinknorpelzellen des Menschen.
}

\author{
Von
}

\section{Shoichi Oinuma.}

Aus dem Anatomischen Institut der Keio Universität, Tokyo.

Mit 17 Textfiguren und 7 Tafelfiguren auf Tafel I.

Inhalt.

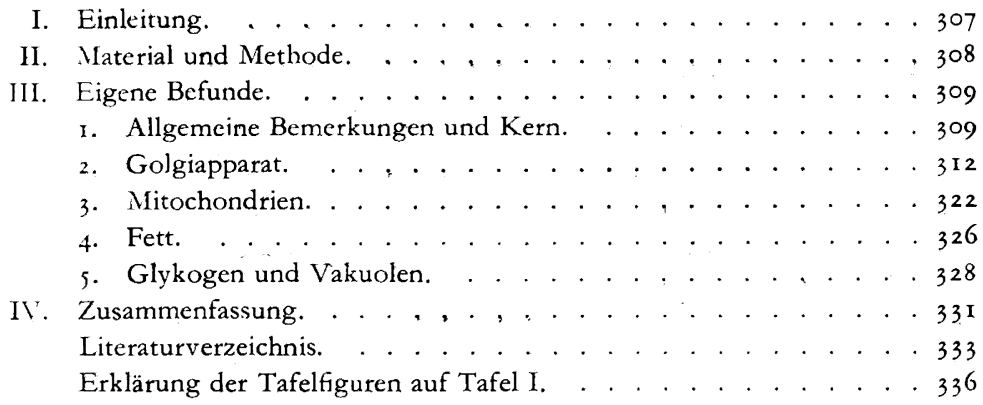

I. Einleitung.

Die Knorpelzellen gehören einer der am häufigsten mikroskopisch untersuchten Zellarten an. Sie sind von zahlreichen Autoren bei Amphibien, Säugetieren, Hühnerembryonen und bei Menschenfeten beschrieben worden. Dabei wurden besonders Kern, Golgiapparat, Vacuome, Mitochondrien, Vakuolen, Fett, Glykogen und Zytozentrum berücksichtigt. Einige Autoren (Fananas (I9I2), Cajal (I9I4), Deineka (IgI6), Fell (I925) u.a.) haben zytologische Veränderungen in den Knorpelzellen, besonders an Golgiapparat und Mitochondrien, bei Ossifikationsprozessen des Hyalinknorpels beschrieben. Solche Veränderungen wurden von Deineka (1916) bei Menschenfeten eingehend angeführt. Dubreuil (I9II) hat auch bei einem Menschenfetus die Mitochondrien in Knorpelzellen beschrieben. Kern, Fett und Glykogen, welche gegen die postmortalen Veränderungen verhältnismässig widerstandfähig sind, wurden bei menschlichen Knorpelzellen relativ häufig untẹsucht, 
doch wurden Golgiapparat und Mitochondrien, welche nach dem Tode schnell zerstört werden, bei menschlichen, wie bei anderen Zellarten, bis heute kaum untersucht. Ich kann hier nur zwei eben referierte Arbeiten von Dubreuil und Deineka über Mitochondrien und Golgiapparat der Hyalinknorpelzellen bei Menschen-Feten angeben. In dieser Hinsicht sind die Knorpelzellen der erwachsenen Menschen bis heute noch unerforscht geblieben. So will ich in der vorliegenden Untersuchung hierzu einige neue Daten beitragen.

\section{Material und Methode.}

Das mir zur Verfügung gestellte Untersuchungsmaterial stammt von dem Trachealknorpel der zwei männlichen Hingerichteten (26 und 36 jähriger), das sofort nach dem Tode lebendfrisch entsprechend dem Zweck in verschiedene Fixierungsflüssigkeiten eingetaucht wurde. Für die Darstellung des Golgiapparates wurde ausschliesslich die Kolatchev'sche Methode benutzt, weil dabei die Fettropfen in Knorpelzellen schwarz osmiert zusammen mit dem Golgiapparat gut konserviert werden. Für den Mitochondriennachweis und das Übersichtspräparat wurden Levi'sche, Regand'sche Flüssigkeit, Formol-Alkohol und 10\% Formol benutzt; die Schnitte wurden mit Heidenhain'schem Eisenhämatoxylin, Anilinfuchsin-Aurantia nach Kull oder Hämatoxylin-Eosin angefärbt.

Das fixierte Material wurde durch die aufsteigende Alkoholreihe entwässert, in Xylol gelegt, in Paraffin eingebettet und dann wurde es in $5 \mu$ dicke Serienschnitte zerlegt. Für die Färbung des Glykogens wurden die durch Formol-Alkohol (95\%) fixierten Zelloidinschnitte, bei welchen vorher Kernfärbung mit Hämatoxylin (Hansen) angestellt war, mit Best'schem Carmin gefärbt. Für die Fettfärbung wurden ausser der Osmiumfärbung nach Kolatchev'scher Methode, die mit 10\% Formalin fixierten Gefrierschnitte mit Sudan III nach Kawamura-Yasaki angefärbt.

Es ist mir eine angenehme Pflicht, hier Herrn Dr. G. Takahashi, Vorstand des pathologischen Institutes des Mantetsu-Hospital zu Hsin-king und Herrn Dr. K. Kiso, Vorstand der Augenklinik desselben Hospital, für ihre freundliche Hilfe bei Ansammlung des Untersuchungsmaterials meinen herzlichsten Dank auszusprechen. 


\section{Eigene Befunde.}

\section{r. Allgemeine Bemerkungen und Kern.}

Wie bekannt, gehört der menschlichen Trachealring zum hyalinen Knorpel, er stellt das ringförmige Gerüst der Trachea dar. Er wird mit einer derben Faserhaut, Perichondrium, umhüllt. In der davon nach innen anschliessenden, ganz hyalin erscheinenden Grundsubstanz sind die Knorpelzellen eingebettet, deren Oberfläche durch eine dünne, stark lichtbrechende Membran, Knorpelkapsel, direkt umgeben ist. Die Gestalt, Grösse und Anordnung der Knorpelzellen in der Grundsubstanz sind, wie allgemein bekannt, nach der Lage verschieden. Im dem Perichondrium angrenzenden peripheren Teil sind die Knorpelzellen klein, spindelförmig oder länglich elliptisch und mit ihrer Längsachse der Oberfläche des Knorpels paralle! liegend vereinzelt verteilt. Nach der Tiefe des Knorpels nehmen sie nach und nach an Grösse zu und werden ebenso allmählich rundlich; nun liegen hier gewöhnlich zwei oder mehr als zwei Knorpelzellen dicht beieinander und stellen eine Zellgruppe dar.

Bei den mit gewöhnlicher Fixierungsflüssigkeit behandelten Paraffinschnitten sieht das Zytoplasma der Knorpelzellen meistens vakuolär aus, was bei den in der Tiefe des Knorpels befindlichen grösseren rundlicher Zellen hochgradig ist. Bei solchen Zellen ist das Zytoplasma durch die verschieden grossen Vakuolen zum grössten Teil besetzt, so dass das Zytoplasma die Zwischenwände der Vakuolen darstellt, welche beim optischen Querschnitt als Zytoplasmastränge zwischen den letzteren erscheinen, wie es bei den Pflanzenzellen häufig der Fall ist. Immer findet man bei solchen Zellen neben dem Kern eine sphärische dichtere Plasmazone, welche granulär oder faserig aussieht und von welcher die Zytoplasmastränge nach der Peripherie etwas radiär ausgehen. Diese dem Kern dicht anliegende Plasmazone ist auch bei den Best's Carmin-Hämatoxylin-Präparaten für Glykogennachweis als ein blass violett gefärbter Plasmabezirk (Tafelfig. 6, 7) und bei den SudanHämatoxylin-Präparaten für Fettfärbung als ein blass rötlich gefärbter (Tafelfig. 3, 4) gut erkennbar. Gleiche Bildung wurde neuerdings von Tretjak off (1927) in den Knorpelzellen der Urodelen als Zentroplasma (Zentrosphäre) beschrieben, welches Diplosom enthält. Seit van der Stricht (1889) wurde Zentrophäre (Zentrotheca) mit Zentriolen von mehrere Autoren bei tierischen Knorpelzellen beschrieben, z. B. von Pensa (I90r), Smirnow (1907), Navikoff (r907), Meves (1910), Fell (1925) u.a., Barinetti (I9r2), Deineka (I9I6) und Dawson (I93I) haben aufmerksam gemacht, dass die Sphäre topographisch mit dem Golgiapparat 
übereinstimmt. Parat et Godin (1925) hat berichtet, dass das Vacuom mit der Zentrosphäre topographisch übereinstimmt.

Nach den Bemerkungen der eben angegebenen Autoren tragen die Knorpelzellen in der Sphäre meistenfalls zwei nebeneinander liegende Zentriolen (Diplosom), doch konnte ich bei den menschlichen Knorpelzellen wie Dawson (193I) in der paranukleären Plasmazone keine Zentriolen nachweisen.

Der Kern der peripheren jüngeren spindelförmigen oder ovalen Zellen ist elliptisch, zuweilen länglich elliptisch gestaltet, er ist verhältnismässig gross. Die Kernmembran ist meistens glatt gespannt. Bei den im Innern des Knorpels gelagerten Zellen ist der Kern mehr rundlich oder oval, zuweilen mehr oder weniger eckig. Im Vergleich zu dem grossen rundlichen Zelleib ist der Kern hier relativ klein, er ist hier meist exzentrisch gelagert. Die Einkerbungen und Furchen sowie dadurch bedingte Lappung des Kerns, welche, wie unten erwähnt, von Autoren oft beschrieben wurden, konnte ich bei den nach Kolatchev behandelten Präparaten, den mit Formol fixierten Gefrierschnitten und den mit Formol-Alkohol fixierten Zelloidinschnitten kaum bemerken. Bei den nach Regand und mit FormolAlkohol fixierten Paraffinschnitten habe ich dagegen zahlreiche hochgradig unregelmässig geformte Kerne vorgefunden. Mir scheint es wahrscheinlich, dass die Unregelmässigkeit der Kernform mindestens zum Teil durch schlechte Behandlung des Untersuchungsmaterials und zwar durch Schrumpfung heryorgerufen wird.

Im Kern findet man in der Regel ein oder zwei, relativ kleine Kernkörperchen; grosse Nukleolen können zuweilen in wechselnder Zahl auftreten. In der Textfig. I4. bemerkt man im Kern einen sehr grossen Nukleolus. In der Textfig. I 3 (links) nimmt ein grosser Nukleolus nahezu den ganzen Kernraum ein. In Textfig. ro und I 2 findet man im Kern je 3 und 4 relativ grosse Nukleolen auf. In seltenen Fällen tragen solch grössere Kernkörperchen im Innern (Textfig. IO) oder auf ihrer Oberfläche osmiophile Granula. In der Textfig. I I bemerkt man, dass zwei dicht nebeneinander liegende, grössere Nukleolen durch osmiophile Substanz gemeinsam umhüllt sind.

Ausser dem Kernkörperchen kommt im Kern selten kleines oder grosses Fettröpfchen vor (Textfig. I5, I6, I7). Noch seltener enthält der Kern Vakuolen (Textfig. Is).

Die Bedeutung des Vorkommens der grossen Nukleolen und Fettröpfchen im Kern wird unten bei der Beschreibung des Golgiapparates näher erörtert. Wir bemerken hier lediglich, dass es sich bei solch grosse Nukleolen führenden Zellen immer um die grossen älteren Knorpelzellen 
handelt, welche in der Zentralpartie des Knorpels zu finden sind.

Novikoff (1907), Fell (1925) u.a. geben bei den Knorpelzellen an, dass der Nukleolus aus dem Kern ins Zytoplasma austreten kann. Saguchi (1928) hat auch bei den Hyalinknorpelzellen der Maus die Emission der geformten Nukleolarsubstanz (argentophile Körnchen) ins Zytoplasma beschrieben, er hat das Schicksal der argentophilen Körnchen erforscht und angegeben, dass sie schlieśslich Knäuel oder Netz bilden und sich zum Golgiapparat entwickeln. Bei den menschlichen Hyalinknorpelzellen konnte ich den Austritt der Nukleolen ins Zytoplasma wirklich nicht bestätigen. Bei den nach Kolatchev behandelten Präparaten bemerkt man oft, wie in den Figuren des Golgiapparates gezeigt, neben dem letzteren kleine kuglige, gleich wie Nukleolus blass braun gefärbte Körnchen. Diese werden aber, wie unten näher beschrieben, mit Sudan III angefärbt, sind also ganz anders als der Nukleolus beschaffen.

Bei den Knorpelzellen der verschiedenen Tiere sind von zahlreichen Autoren die Mitose und Amitose zugleich mit der Mehrkernigkeit beschrieben worden. Neuerdings wurden sie von Fler off (I 929/30) sehr eingehend erklärt, und man kann dort ein näheres Literaturverzeichnis finden. Die direkte Teilung der Knorpelzellen ist von zahlreichen Autoren angegeben worden (Bütschli (I877), Bigelow (1879), Hammer (1894), Studnicka (1897), Schaffer (r90I), Hansen (1905), Fleroff (1924), Tretjak off (1927) u. a.). Die Mehrzahl der eben genannten Autoren bemerkte die Lappung oder Abschnürung des Kerns (s. oben), und gibt damit die direkte Teilung der Knorpelzellen an. Nagamatsu (1924) hat bei den Knorpelzellen verschiedene Formen des Kerns zwar bemerkt, doch hat er gegenüber den anderen Autoren das Vorkommen der amitotischen Kernteilung negiert. Anderseits ist die indirekte Teilung bei den Knorpelzellen auch von ebenso zahlreichen Autoren angegeben worden (Flemming (i882), van der Stricht (1887, '92), Sieveking (1892), Fell (1925) u.a.). Flemming hat behauptet, dass die Karyokinese die einzige mögliche Kernteilungsform der Knorpelzellen sei. Nawik off (1909), Deineka (1916), Fle roff (1929/30) u.a. geben das Vorkommen der Amitose als auch der Mitose an. Nach Navikoff (1909) vermehren sich die Zellen des eben gebildeten jungen Knorpels ausschliesslich karyokinetisch. Bei altem Knorpel erscheint die amitotische Teilung und spielt nun eine grössere Rolle als die Karyokinese. Diese Angabe Navik offs scheint mir sehr zutreffend zu sein. Die Untersuchungsmaterialien der Autoren, welche bei den Knorpelzellen die karyokinetische Teilung bemerkt haben, stammen alle von dem Embryo oder den verschiedenen jüngeren Tieren; Flemming hat die Knorpel von Salamanderlarve, Deineka die von Embryo der verschiedenen Säugetiere 
und Fell die von Hühnerembryo benutzt.

In der vorliegenden Untersuchung des menschlichen Trachealknorpels habe ich weder die mitotische, noch die amitotische Teilung der Knorpelzellen wahrgenommen. Wie oben erwähnt, habe ich bei den KolatchevPräparaten u.a. keine ausgeprägte lappige oder abgeschnürte Kerne bemerkt, welche nach Autoren auf das Vorkommen der amitotische Teilung des Kerns hindeuten. Die unregelmässigen Gestalten des Kerns in den Regaud- und Formol-Alkohol-Paraffinschnitten halte ich für Kunstprodukte.

Unter den Knorpelzellen des menschlichen Trachealknorpels habe ich aber selten die zweikernigen gefunden (Textfig. 7). Die zweikernige Zellen traf ich immer bei den grossen rundlichen Zellen, aber niemals bei den oberflächlichen spindelförmigen an. Die zwei Kerne liegen nahe nebeneinander, sind nahezu gleichgross. Die Mehrkernigkeit der Knorpelzellen ist auch bei verschiedenen Tierarten oftmals zusammen mit der Kernteilung von zahlreichen Autoren beschrieben worden, z.B. von Leidy (1849), Heidenhain (1863), Hannover (1865), Flemming (1882). Navikoff (1909) Pensa (1913), Deineka (1916), Fell (1925), Tretjak off (1929), Fleroff (1929/30), Dawson (1931), Murata (1933 a) u.a. Die meisten Autoren beschreiben nur zweikernige Knorpelzellen, während Ha n nover (1865) und Tretjak off (1927) sogar über dreikernige berichteten. Als Ursache der Mehrkernigkeit der Knorpelzellen hat Flemming (1882) die Abortivmitose angegeben, bei welcher es sich um eine ohne Achromatinspindel verlaufende Karyokinese handelt. Diese Angabe wurde neuerdings bei den Knorpelzellen von Fle roff (1929/30) bestätigt. Demgegenüber haben Flem ming und Fle r of $f$ Navikoff (1909), Tretjak off (1927) u. a. angegeben, dass die Mehrkernigkeit der Knorpelzellen infolge der Amitose bedingt werden.

Ich kann in der vorliegenden Untersuchung keine entschiedene Antwort über diese Frage geben, aber werde auf diese bei der Beschreibung des Golgiapparates noch einmal zurückkommen. Wie oben beschrieben, kommt Zweikernigkeit bei dem menschlichen Trachealknorpel nicht an den spindelförmigen jungen Zellen, sondern nur an den tieferen rundlichen alten Zellen vor; daraus kann man wohl schliessen, dass im Trachealring des erwachsenen Menschen entweder Amitose oder Mitose ohne Begleitung der Teilung des Zelleibes wirklich stattfindet, obwohl ich sie nicht nachweisen konnte. Nach meiner Vermutung handelt es sich dabei vielmehr um Amitose.

\section{Golgiapparat.}

Der Golgiapparat der Knorpelzellen wurde seit Pensa (I90I) und Bergen (1904) von recht zahlreichen Autoren beschrieben. Pensa hat im Zytoplasma der Rippenknorpelzellen der Katze einen Netzapparat aus 
verästalnden, ineinander verflochtenen Fäden nachgewiesen und als ein dem von Golgi kurz vorher entdeckten Binnennetzapparat analoges Gebilde bezeichnet. Der im Jahre 190 I von Pensa demonstrierte Netzapparat der Knorpelzellen wurde späterhin von Meves (1907), Comes (1909) und von Pensa (1913) selbst mit den Mitochondrien identifiziert; so denkt man heute, dass der erste Entdecker des Golgiapparates der Knorpelzellen Bergen ist. Seit den genannten beiden Autoren wurde der Golgiapparat

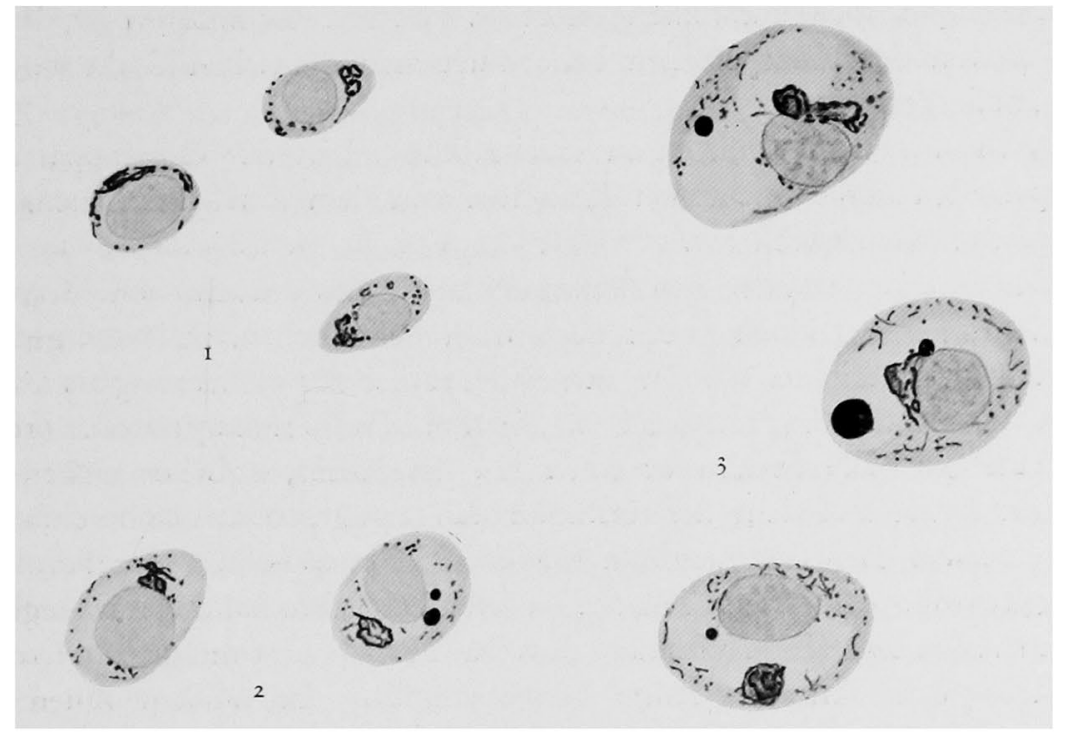

Textfig. I-3. Knorpelzellen aus der oberflächlicheren Schicht des Trachealknorpels, Mensch. Kol a tchev'sche Osmiumimprägnationsmethode. Zeiss Apochr.-Imm. 2 mm, n. A. I.3, Komp.-Ok. I 2.

der Knorpelzellen von einer ganzen Reihe Autoren bei verschiedenen Tierarten untersucht, so z. B. von Smirnow (1907), Comes (1909), Duesberg (I9II), Barinetti (I9I2), Fananas (I9I2), Comes (1913), Pensa (1913), Cajal (1914), Kolmer (1915), Deineka (1916), Fell (1925), Parat et Godin (1925), Tretjak off (1927, '28), Saguchi (1928), Parat (1928), Tanaka (1928), Dawson (I93I) und Murata (1633a,b), unter denen Fananas, Cajal und Deineka auch die Veränderung des Golgiapparates der Knorpelzellen bei der Ossifikation näher verfolgt haben.

Nach den Ergebnissen der eben genannten Forscher stellt der Golgiapparat der Knorpelzellen der verschiedenen Säugetiere, Amphibien und des Hühnerembryos meistenfalls ein neben dem Kern liegendes kleines Netz, Knäuel oder Klümpchen dar. Nach Tretjakoff (1927, '28) bildet der Golgiapparat bei Amphibien nicht ein umschriebenes Knäuel aus, sondern 
die Golgielemente verstreuen sich als Stäbchen oder Körnchen im ganzen Zelleib. Nach Dawson (193I) stellen die Golgielemente bei Necturüs zuweilen verstreute Fäden dar. Nach Comes stellt der Golgiapparat ein im ganzen Zelleib ausbreitendes Netz dar. Unter den genannten Autoren hat nur Deineka den Golgiapparat der Knorpelzellen beim Menschenembryo untersucht. Nach seiner Angabe ist der Golgiapparat der jungen Knorpelzellen ein neben dem Kern liegendes Körperchen, welches aus gebogenen Fäden (Dictyosomen) zusammengesetzt ist. Soweit mir bekannt ist, ist der Golgiapparat der Knorpelzellen bei erwachsenen Menschen bis heute noch nicht studiert worden.

Nach meinen Ergebnissen an Trachealknorpel ist der Golgiapparat der Knorpelzellen der erwachsenen Menschen von dem des Menschenembryos wesentlich nicht verschieden.

Der Golgiapparat der Trachealknorpelzellen des erwachsenen Menschen stellt in der Regel einen neben dem Kern befindlichen, meistens gut umschriebenen rundlichen Körper dar, welcher bei der Osmierung als Ganzes schwach schwarz tingiert wird (s. Figuren des Golgiapparates). In der Mehrzahl der Fälle berührt er einen Pol des Kerns, wobei er nicht selten mit einer breiteren Fläche der Kernmembran anliegt, so dass er bei Seitenansicht eine auf der Kernmembran sitzende Halbmond- oder Kegelform aufweist (Textfig. 3 (mittler), Textfig. 6). Ich habe aber bei den menschlichen Knorpelzellen niemals angefunden, dass der Golgiapparat den ganzen Umfang des Kerns oder ihn über halbe Länge umgibt. Er umfasst ihn immer unter halber Länge des Umfanges, wie Bergen (I904), Deineka(r9i6) u. a. aufmerksam gemacht haben. Zuweilen sind der Kern und der Gol-
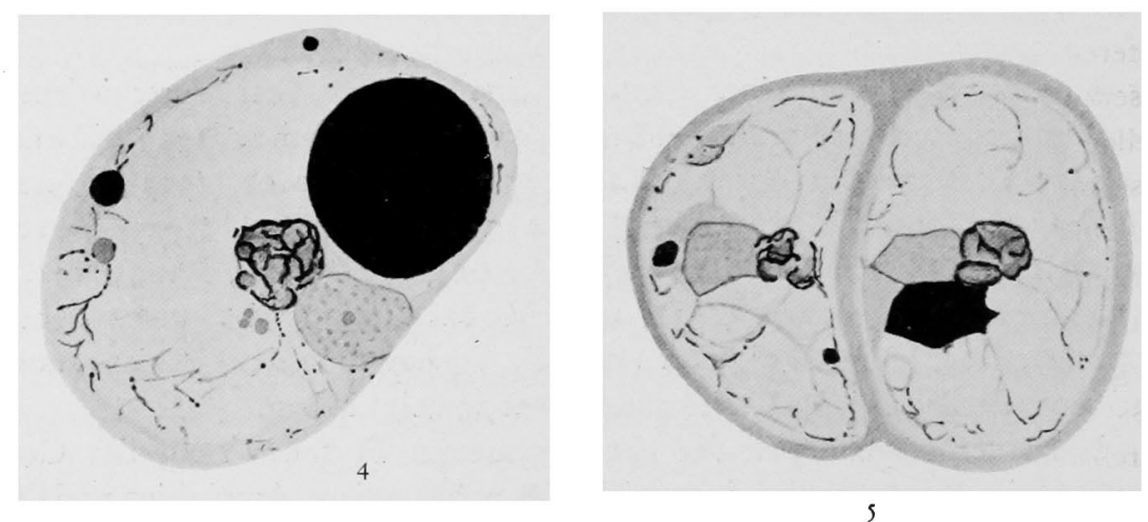

Textfig. 4-5. Knorpelzellen aus der tieferen Schicht des Trachealknorpels, Mensch. Kolatchevsche Osmiumimprägnationsmethode. Zeiss Apochr.-Imm. $2 \mathrm{~mm}$, n. A. 1.3, Komp.-Ok. 12. 
giapparat voneinander ein wenig entfernt, selten ist der Abstand zwischen beiden Gebilden ziemlich weit (Textfig. 3 (unten)).

Was die Grösse des Golgiapparates der einzelnen Knorpelzellen angeht, so geht sie gewöhnlich mit der Grösse der Zellen parallel; sie ist bei den kleinen Zellen der Randzone des Knorpels klein (Textfig. I, 2), aber bei den grossen der tieferen Zone gross (Textfig. 3, 4, 5, 6). Sie überschreitet niemals die Kerngrösse, wie von Deineka (1916) Murata (1933a) u. a. berichtet. Die Grösse des Golgiapparates variiert ausserdem nach dem Zustand der einzelnen Zellen im gewissem Masse (s. unten).

Betrachtet man den Golgiapparat mit stärkerer Vergrösserung näher, so kann man seine Innenstruktur beobachten. Er besteht immer aus tief schwarz imprägnierten Fäden oder Strängen, welche geknickt verlaufend miteinander kompliziert anastomosieren. Also stellt der Golgiapparat der Knorpelzellen des erwachsenen Menschen ein ziemlich dicht gebautes, engmaschiges Netz oder Knäuel dar, welches, wie oben bemerkt, in der Regel gut umschrieben und allseitig geschlossen ist. So stimmt mein Befund mit dem von Bergen (1904), Cajal (19i4), Kolmer (1915), Deineka (1916), Saguchi (1928), Murata (1933) u. a. bei den Säugetieren und dem Menschenfetus im grossen ganzen überein. Wie bei den anderen Zellarten sind die Stränge des Golgiapparates der Knorpelzellen nicht gleichmässig gebaut, sondern sie tragen mehr oder weniger gut ausgeprägte Verdickungen, wie von Deineka (1916) u. a. bei den Knorpelzellen berichtet worden ist.

Diese Netzstruktur ist nach den Zellen der Randzone und der tieferen Schicht des Knorpels im Wesentlichen nicht verschieden; der Golgiapparat ist in den Zellen der Randzone klein, doch ist er, wie in den Zellen der tieferen Schicht, aus geknickten und anastomosierenden Fäden zusammengesetzt (Textfig. I, 2). Nach Fell (1925) sieht der Golgiapparat in den Zellen von gewisser Lokalisation wie eine lockere Anhäufung von Stäbchen aus, und nach Murata (I9I3a) sind die Elemente des Golgiapparates in den Zellen der Randzone des Knorpels Granula. Diese Befunde konnte ich bei dem menschlichen Trachealknorpel nicht bestätigen. Bergen (1904), Murata (1931) u. a. haben bemerkt, dass die Stränge oft von dem Apparat in gewisser Strecke frei ausgehen. Saguchi (1928) hat sogar angegeben, dass die Stränge in einzelne Stückchen zerfallen und in das Zytoplasma durchwandern, um die Zellgrenze zu überschreiten und in die Grundsubstanz einzudringen. Diese Befunde konnte ich jedoch niemals bestätigen. Nach meiner Beobachtung ist der Golgiapparat meistens geschlossen, und entsendet nicht die freien Stränge nach aussen hin.

Die Frage, wie verhält sich der Golgiapparat in der zweikernigen Knor- 
pelzelle, wurde von einigen Autoren (Pensa (1913), Dawson (1931) und Murata (1933)) auseinandergesetzt. Bei Pensa's Abbildungen versteht man, dass der Golgiapparat entweder zwischen zwei Kernen vorhanden ist oder auf einer Seite der beiden Kerne diese brückenartig überbrückt. Nach Daw son und Murata ist der Golgiapparat bei den zweikernigen Knorpelzellen zwischen zwei Kernen vorhanden. Also kommt der Golgiapparat bei zweikernigen Knorpelzellen nur einfach vor und ist den zwei Kernen gemein. Deineka (1912) hat bei der Untersuchung des Golgiapparates der Epithelund Bindegewebszellen entdeckt, dass der Golgiapparat bei der Mitose ebenso zweigeteilt wird, so dass die Tochterkerne je einen Golgiapparat besitzen, während bei der Amitose der Golgiapparat nicht geteilt wird, so dass er von zwei Kernen gemeinsam eingenommen wird. Später hat er (1916) auch bei der Untersuchung der Knorpelzellen darauf aufmerksam gemacht, dass bei der Mitose der Knorpelzelle der Golgiapparat sich auch in zwei teilt. Von der Angabe Deineka's geschlossen, soll die Zweikernigkeit der Knorpelzellen infolge der Amitose entstehen, weil nach den Ergebnissen der oben genannten Autoren bei den zweikernigen Knorpelzellen der Golgiapparat ungeteilt bleibt.

Wie oben erwähnt, konnte ich auch bei dem menschlichen Trachealknorpel selten zweikernige Knorpelzellen wahrnehmen. Ich habe bei solchen zweikernigen Zellen gefunden, dass der Golgiapparat nicht vollkommen zweigeteilt, aber lang gestreckt auf einer Seite der Kerne zwischen diesen überbrückt ist (Textfig. 7). Er zeigt aber die Andeutung der Zweiteilung, indem er sich in der Mitte etwas verjüngt, die Golgielemente sich an beiden Enden, wo der Golgiapparat den Kern berüht, ansammeln und je ein Knäuel bilden. Ich konnte bei den menschlichen Knorpelzellen nicht den Zustand begegnen, bei dem der Golgiapparat zwischen zwei Kernen einfach vorkommt. Also konnte ich nur den Pensa's zweiten Befund bestätigen. Aus obigen Befunden des Golgiapparates darf ich annehmen, dass der Golgiapparat, falls die zweikernige Knorpelzelle sich nicht in zwei Tochterzellen teilt und zweikernig bleibt, ebenso ungeteilt und fortwährend den eben genannten Zustand erhält; falls die Teilung der zweikernigen Zelle stattfindet, so wird der gestreckte Golgiapparat auch an der verjüngten Stelle zweigeteilt und geht in einzelne Tochterzelle über. Nach Deineka (I912, 'I6) zerfällt der Golgiapparat bei der Mitose zuerst in zahlreiche Golgielemente (Dictyosomen), deren ungefähre Halbzahl sich je an beiden Spindelpolen ansammelt, um dort schliesslich je einen Golgiapparat der Tochterzelle zu bilden. Ich konnte bei den Knorpelzellen des menschlichen Trachealringes niemals die Zerteilung des Golgiapparates in einzelne Dictyosomen wahrnehmen. Daraus kann das Vorkommen 
der Mitose in den menschlichen Trachealknorpelzellen wahrscheinlich ausgeschlossen werden. Wie oben erwähnt (S. 3 I 2), geben Autoren bezüglich der Zweikernigkeit und der Teilung der Knorpelzellen einerseits die Amitose, anderseits Mitose an. Ich darf nun behaupten, dass die Teilung und die Zweikernigkeit mindestens bei den erwachsenen menschlichen Trachealknorpelzellen immer durch Amitose erfolgen.

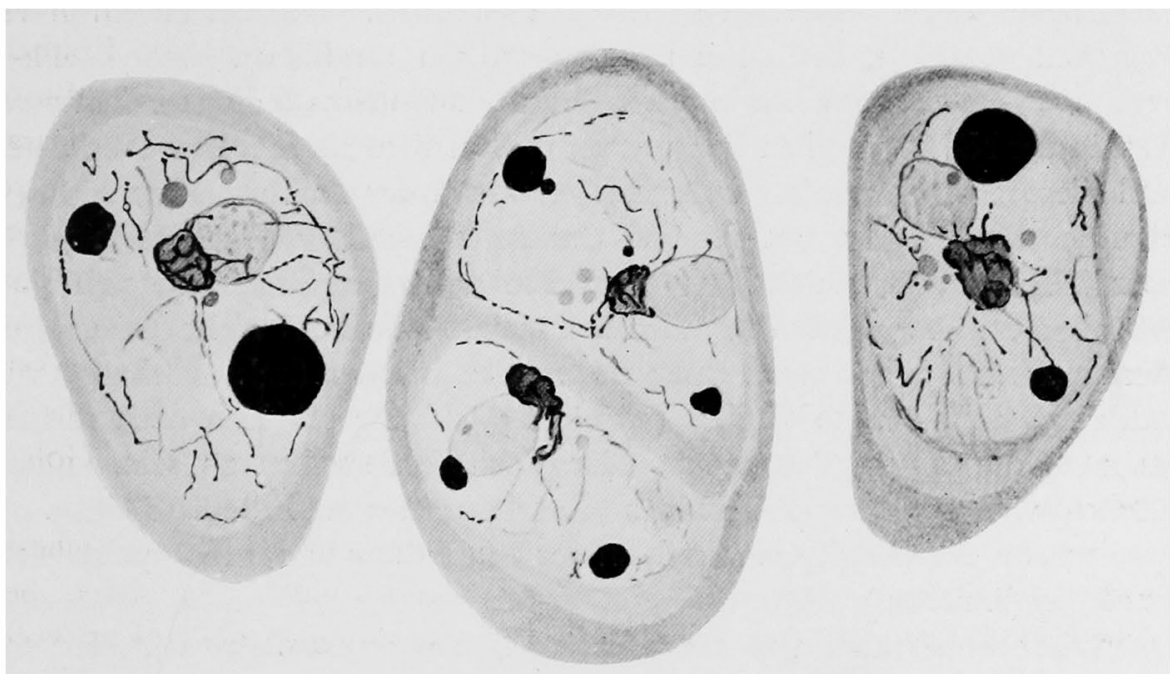

Textfig 6. Knorpelzellen aus der tieferen Schicht des Trachealknorpels, Mensch. Kolatchevsche Osmiumimprägnationsmethode. Zeiss Apochr.-Imm. $2 \mathrm{~mm}$, n. A. I.3, Komp.-Ok. I 2.

Ich habe oben dargelegt, dass in den Knorpelzellen des menschlichen Trachealringes neben dem Kern eine sphärische dichtere Plasmazone vorhanden ist, welche dem Zentroplasma oder der Zentrosphäre der Autoren entspricht (s. S. 23I). Die topographische Übereinstimmung der Zentrosphäre und des Golgiapparates bei den Knorpelzellen ist von Autoren, wie Barinetti (I9I2), Deineka (I9I6), Dawson (I93I) und Parat et Godin (1925), angegeben worden. Diese Angabe konnte ich bei den menschlichen Knorpelzellen bestätigen. Für diese Bestätigung habe ich folgendes Verfahren durchgeführt. Die Kolatchev-Präparate werden zunächst mit Rubaschkin'scher Methode gebleicht, bis der Golgiapparat der Knorpelzellen vollständig entfärbt ist. Dann werden die gebleichten Präparate nach Kull mit Anilinfuchsin-Aurantia gefärbt und mikroskopiert. Ich konnte dabei in den Knorpelzellen an Stelle des Golgiapparates neben dem Kern eine dichtere Plasmazone auffinden, welche der bei den gewöhnlichen Präparaten und bei den Sudan-Hämatoxylin- sowie Best's Carmin-Hämatoxylin- 
Präparaten bemerkbaren Plasmazone gänzlich entspricht. Bei den Kolatchev-Präparaten kann man solche Plasmazone nicht wahrnehmen; so liegt es nahe, dass sie durch den Golgiapparat vollkommen besetzt ist. Also konnte ich bei den menschlichen Knorpelzell $n$ feststellen, dass die Golgizone mit der von Autoren als Zentrosphäre bezeichneten Plasmazone gänzlich übereinstimmt. Ich konnte aber, wie oben erwähnt, in dieser paranuklearen Plasmazone keine Zentriolen nachweisen (s. oben). Doch habe ich in der Mehrzahl der Fälle, besonders bei den grossen rundlichen Knorpelzellen, kreisrunde kleine Körnchen in wechselnder Zahl innerhalb dieser Plasmazone oder in der Nähe derselben vorgefunden. Bei den Kolatchev-Präparaten sind diese Körnchen blass bräunlich wie etwa der Nukleolus angefärbt (s. Figuren des Golgiapparates). Bei der Anilinfuchsin-Färbung nach Kull oder Eisenhämatoxylin-Färbung nach Heidenhain färben sie sich gut. Wie oben erwähnt, sind diese Körnchen meines Erachtens nicht die aus dem Kern stammte Nukleolarsubstanz; sie werden bei der Sudan-Färbung, wie unten erwähnt, orange tingiert (Tafelfig. 3, 4). Sie scheinen nach meiner Meinung die jungen Fettgranula oder Vorstufe derselben zu sein. Diese Körnchen sind oft innerhalb des Golgiapparates enthalten (Textfig. 4). Also scheint der Golgiapparat sich an der Fettbildung in der Knorpelzelle zu beteiligen (s. unten.)

Wie schon früher von Bergen (1904) aufmerksam gemacht, ist der Golgiapparat der Knorpelzellen mit demselben der anderen Bindesubstanzgewebezellen analog, indem er meistenfalls auf einem Pol des Kerns ein gut umschriebenes Netzkörperchen oder Knäuel darstellt. So kann man wohl sagen, dass bei den Knorpelzellen der Golgiapparat polar gelagert ist. Die Richtung des Golgipols der Knorpelzellen bzw. die Lagebeziehung solch polar gelagertes Golgiapparates gegenüber der Oberfläche des Knorpels wurde zuerst von Saguchi (1928) untersucht. Nach seiner Beobachtung ist der Golgiapparat meistens im nach dem Zentrum des Knorpels zugekehrten Teil des Zelleibes gelagert; der Kern ist über dem Golgiapparat gelegen. Im mittleren Teil des Knorpels ist der Golgiapparat aber seitwärts vom Kern gelegen. Er hat dabei die „, Kern-Knäuel-Linie “ vorgeschlagen, welche von der durch Kernmitte und Knäuel(Golgiapparat-)mitte ziehende Gerade repräsentiert wird. Im ersteren Fall ist diese Linie zur Oberfläche des Knorpels senkrecht, im letzteren steht sie dazu parallel. Nach Saguchi zeigt die charakteristische Richtung des Golgipols der Knorpelzellen die Richtung der Abgabe der ernährenden Substanz von Knorpelzellen zu Grundsubstanz. Dann wurde weiter die Lagebeziehung des Golgiapparates bei den Knorpelzellen von Dawson (1931) und Murata (1933a) eingehend auseinandergesetzt. Die Ergebnisse beider Autoren stimmen nahezu 
überein. Nach beiden Autoren ist der Golgiapparat in den spindelförmigen Knorpelzellen der Randzone des Knorpels meistens in einer Seite des Kerns gelegen, in den noch tiefer gelagerten rundlichen Zellen liegt er auf der tieferen Seite des Kerns und in den in der Mittelpartie des Knorpels befindlichen Zellen ist die Lage des Golgiapparates unregelmässig. Murata (1933b) hat weiter diese Frage experimentell studiert.

Nach meiner eigenen Beobachtung bei dem menschlichen Trachealknorpel ist die Lage des

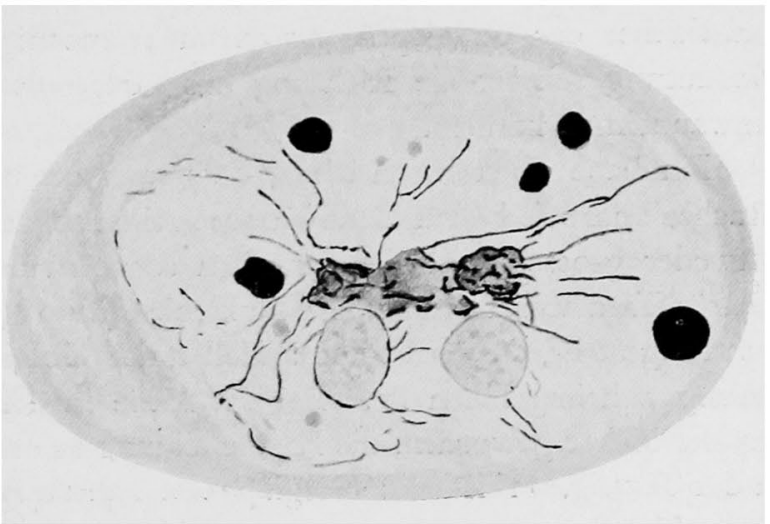

Textfig. 7. Zweikernige Knorpelzelle aus der tieferen Schicht des Trachealknorpels, Mensch. Kolatchersche Osmiumimprägnationsmethode. Zeiss Apochr.-Imm. $2 \mathrm{~mm}$, n. A. I.3, Komp.-Ok. I 2. Golgiapparates in den spindelförmigen oder länglich ovalen Zellen seitwärts vom Kern zu finden, wie von Dawson und Murata angegeben. Also steht die Kern-KnäuelLinie nahezu parallel der Oberfläche des Knorpels. Bei den nach der Tiefe des Knorpels anschliessenden rundlichen Knorpelzellen ist die Lage des Golgiapparates gegenüber der Knorpeloberfläche schon regellos, indem der Apparat einmal in der tieferen Seite des Kerns, andermal in der oberflächlichen Seite oder seitlich des Kerns lokalisiert ist. In der Zentralpartie des Knorpels ist die Richtung des Golgipols ebenso regellos. Also scheint dem menschlichen Trachealknorpel die Zone, wo die Richtung des Golgipols regelmässig nach der Tiefe des Knorpels gerichtet ist, wie von $\mathrm{Daw}$ åon und Murata angegeben, zu fehlen.

Bei den paarig eine Gruppe bildenden Knorpelzellen ist der Golgiapparat nach Dawson (1931) in der nach der anderen Zelle gerichteten Seite des Kerns gelagert, nach Murata (1933) liegt der Golgiapparat meistens in der umgekehrten Seite der beiden Kerne. Nach ihm entspricht der Befund Dawson's dem speziellen Fall des seinigen. Ich habe in der vorliegenden Untersuchung die Befunde der beiden Autoren bestätigt. Einmal habe ich bei den parigen Zellen an dem nach der anderen Zelle zugekehrten Pol des Kerns (Textfig. 6 (mittler)), andermal am umgekehrten Pol der beiden Kerne den Golgiapparat gefunden. Weiter habe ich das in der Textfig. \& wiedergegebene Verhältnis gefunden, wobei der Golgiappa- 
rat bei der einen Zellen in der der anderen Zelle zugekehrten Seite des Kerns, bei der anderen Zelle in der der anderen Zelle gegenüberstehenden gelegen ist. Wir können somit sagen, dass bei den paarigen Zellen die Lageverhältnisse des Golgiapparates vielmehr mannigfaltig sind. Nach meiner Vermutung scheint die Richtung des Golgipols der einzelnen Zellen sich umwandeln zu können.

Ich habe oben beschrieben, dass man oft unter den im Zentralteil des Trachealknorpels befindlichen Knorpelzellen solche auffindet, welche im Kern entweder grosse Nukleolen zuweilen sogar mehrfach enthalten oder seiten Fettröpfchen ausser den grossen Nukleolen führen (Textfig. 10-17). Ich habe nun festgestellt, dass bei solchen Zellen der Golgiapparat meistens in Veränderung anheimgefallen ist. Bei der Zelle in der Textfig. Io ist der Golgiapparat locker geworden, die Knäuelbildung ist etwas zerstört. Bei der Zelle in der Textfig. II ist der Golgiapparat nahezu normal ; er ist bei der Zelle in der Textfig. I2, welche 4 grosse Nukleolen enthält, verkleinert und seine Knäuelbildung ist sehr einfach. Die in der Textfig. I 3 links abgebildete Zelle trägt einen ausserordentlich grossen nahezu den ganzen Kernraum einnehmenden Nucleolus. Der Golgiapparat stellt dabei einen relativ grossen Körper dar, bei welchem aber die das Knäuel bildenden Golgielemente dünn geworden sind und ihre Osmierbarkeit in hohem Masse reduziert ist. Man findet in der rechten Zelle einen normalen Golgiapparat. Also kann man leicht verstehen, dass die Veränderung des Golgiapparates in der linken Zelle nicht auf einen Technikfehler zurückzuführen ist. In der auf der Textfig.
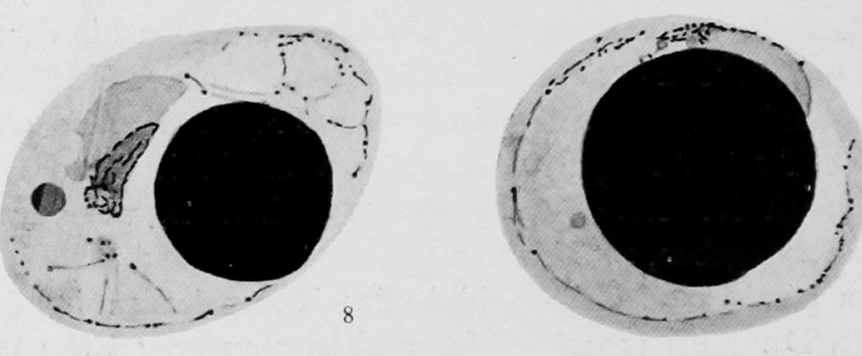

9

Textfig. 8-9. Die von einem grossen Fettropfen gefüllten Knorpelzellen aus der tieferen Schicht des Trachealknorpels, Mensch. Kolatchersche Osmiumimprägnationsmethode. Zeiss Apochr.-Imm. 2 mm, n. A. 1.3, Komp.-Ok. $\quad 12$.

I4 wiedergegebenen Zelle ist der Golgiapparat auf einen kleinen, dem einen Pol des Kerns anliegenden Körper reduziert, worin nur wenige feine Golgistränge bemerkbar sind. Die auf der Textfig. Is rechts abgebildete Knorpelzelle führt einen grossen Kern, welcher grobgranulär ist und ausser den 
grossen Nukleolen kleine Fettröpfchen und eine Vakuole enthält. Dass dieser Kern hochgradig verändert ist, kann man ohne wciteres ersehen. Der Golgiapparat stellt ebenso einen kleinen dunklen Körper dar, worin man kaum die Netzstruktur findet. Bei dieser Zelle findet man in Zytoplasmasträngen des Zelleibes keine osmierte Fäden (Mitochondrien), der Zelleib sieht ganz blasig aus. Die links liegende Knorpelzelle ist normal. Die auf der Textfig. 16 und 17 abgebildeten, ebenso blasigen Knorpelzellen führen ebenso ausserordentlich grossen und grobgranulierten Kern, welcher ausser den grossen Nukleolen ausserordentlich grossen, sogar den ganzen Kern besetzenden (Textfig. 16) Fettropfen enthält. Bei diesen Zellen findet man nicht mehr den Golgiapparat. Bei der auf der Textfig. 16 abgebildeten Zelle findet man von dem Kern entfernt Klümpchen der schwach osmierten Granula, welche scheinbar dem zerstörten Golgiapparat dieser Zelle entsprechen. Nach den Beschaffenkeiten des Golgiapparates müssen die oben erwähnten Knorpelzellen die nach und nach rückbildenden Knorpelzellen sein. Es ist dabei sehr beachtenswert, dass bei solchen degenerierenden Zellen die Nukleolen an Grösse und an Zahl, somit an Menge zugenommen
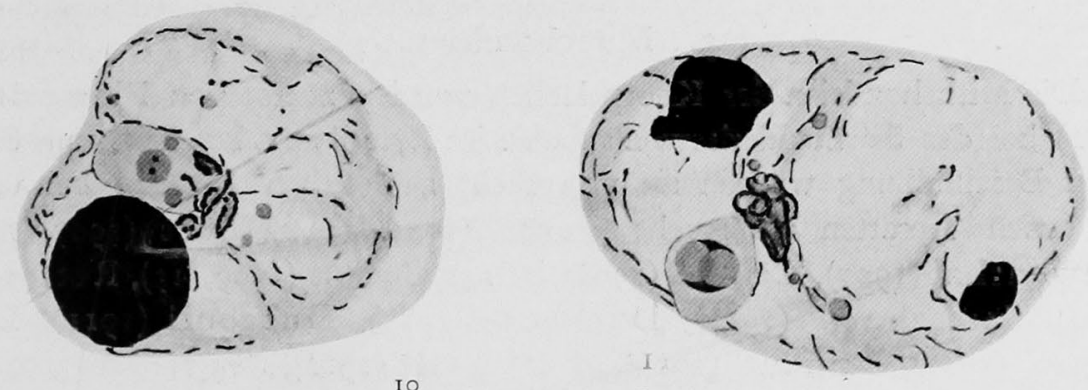

IO
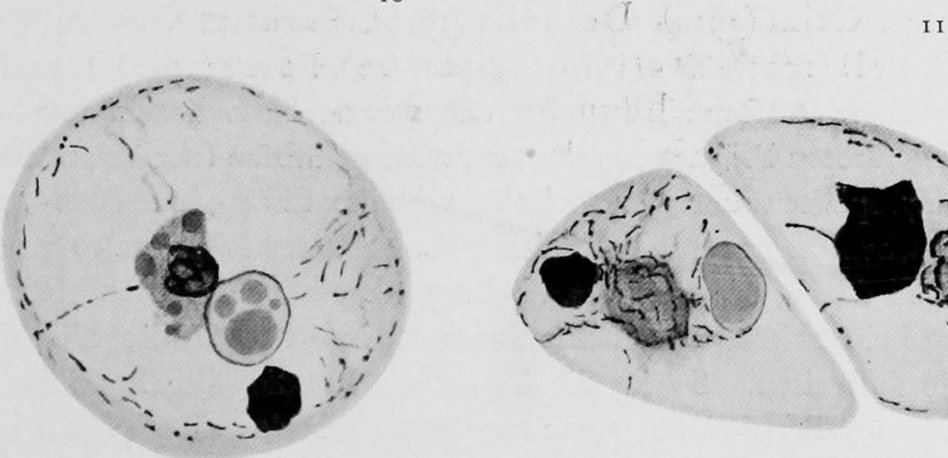

I 2

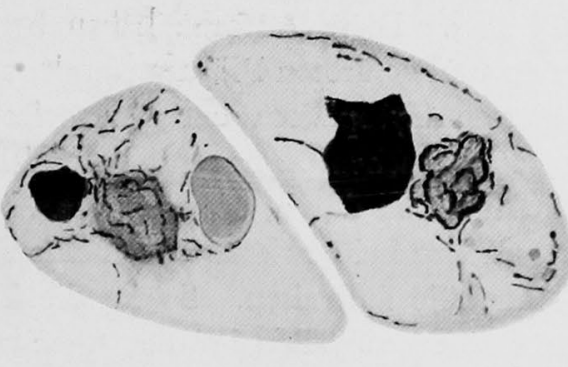

I 3

Textfig. 10-13. Knorpelzellen aus der tieferen Schicht des Trachealknorpels, Mensch. Kolatchevsche Osmiumimprägnationsmethode. Die Kerne enthalten alle grosse Nukleolen. Zeiss Apochr.-Imm. 2 mm, n. A. I.3, Komp.-Ok. 12. 
sind. Der Golgiaparat selbst ist ebenso rückgebildet, indem zuerst die Golgistränge dünner und schwächer werden, dann der Apparat im Ganzen sich verkleinert und schliesslich zerstört und gänzlich verschwindet. Im Endstadium der Rückbildung vergrössert sich der Kern in hohem Masse, wird grobgranuliert und es erscheinen darin Fettröpfen. Wie oben gesagt, werden diese degenerierenden Zellen im Zentralteil des. Knorpels gefunden; dort gehen also veraltete Knorpelzellen unter allmählicher Degeneration zugrunde.

Ausserdem findet man die Rückbildung des Golgiapparates bei den mit sehr grossen Fettropfen, wie bei den Fettzellen, stark gefüllten Knorpelzellen, bei welchen, wie in der Textfig. 8 und 9 wiedergegeben, der Golgiapparat zusammen mit dem Kern nach einer Seite des Zellkörpers stark verdrängt wird und halbmondförmig erscheint. In der Tat verkleinert er sich und seine Stränge sind in hohem Masse feiner geworden. An dem Kern findet man aber keine Veränderung; die Rückbildung des Golgiapparates sei bei diesem Fall höchstwahrscheinlich infolge Abdrücken durch Fettropfen hervorgerufen worden.

\section{Mitochondrien.}

Die Mitochondrien der Knorpelzellen wurden zuerst von Flemming (1882) bei der Salamanderlarve als Fäden im Zytoplasma beschrieben. Seit seiner Beschreibung wurden sie yon recht zahlreichen Autoren bei verschiedenen Tierarten untersucht (Pensa (1901, '13), Meves (1907, '10), Loewenthal (1907), Arnold (1908a, b, '14), Comes (I909,'13), Retterer (1909), Samssonow (rgro), Duesberg (igro), Dubreuil (r9ri), Laguesse (I9II), Renaut (I9II), Barinetti (I9I2), Torraco (I9r4), Retzius (1914), Cajal (1914), Deineka (1916), Parat et Godin (1925), Lams (1925), Fell (1925), Tretjak off (1927, '28), Parat (1928), Dawson (1931) u.a.). Diese Autoren haben bei den mit der Mitochondrialmethode fixierten und gefärbten Präparaten oder supravital gefärbten frischen, meistens im ganzen Zytoplasma der Knorpelzelle verteilte faden-, stäbchen- oder zuweilen körnchenförmige Mitochondrien beobachtet. Liest man die zahlreiche Literatur durch, so wird man belehrt, dass die Mitochondrien der Knorpelzellen bei gewissen Fällen eine ungemein intensive Osmiophilie oder Argentophilie aufweisen. So ist die Tatsache, dass die Mitochondrien der Knorpelzellen zuweilen bei der Osmium- oder Silberimpränation zur Darstellung des Golgiapparates zusammen mit dem letzteren schwarz angefärbt werden können, von Pensa (1901, 'I3), Comes (1909, 'I3), Deineka (1916), Tretjakoff (1928), Dawson (1931) u.a. berichtet worden. Obwohl nicht selten von Autoren auf die gleiche Tatsache bei anderen Zellarten auf- 
merksam gemacht worden ist, scheint die Imprägnierbarkeit bei den Knorpelzellen besonders intensiv und regelmässig zu sein. Wegen dieser besonderen Eigenschaft der Mitochondrien hielt Pensa (I901) zunächst bei den Knorpelzellen des Rippenknorpels der erwachsenen Katze die mit Silbernitrat schwarz imprägnierten Mitochondrien für Golgiapparat, obwohl er dann (1913) seinen Fehler korrigierte. Comes (I909, 'I3) zog sogar den Schluss, dass bei den Knorpelzellen das Chondriom und der Golgiapparat identische Gebilde seien, da er bei der Benda'schen Mitochondrialmethode in den Zellen des Rippenknorpels der Maus vollkommen gleiche Netze, wie mit der Golgi'schen Silberimprägnationsmethodé, neben diesen aber keine andere mitochondrienähnliche Gebilde darstellen konnte. Neuerdings wurden diese Imprägnationsverhältnisse der Mitochondrien in Knorpelzellen von Deineka (1916) und Dawson (193I) eingehend beschrieben, nach Dawson sind die Mitochondrien und Golgielemente oft schwer unterscheidbar. Deineka hat aber merkliche Unterschiede zwischen den beiden Bildungen angegeben, wie unten referiert.

Ich habe auch in der vorliegenden Untersuchung bei dem menschlichen Trachealknorpel die Angabe der Autoren bestätigt, dass die Mitochondrien der Knorpelzellen bei der Osmierung prächtig schwarz, wie der Golgiapparat, imprägniert werden. In den Kolatchev-Präparaten, bei welchen die Imprägnation ideal erfolgt ist, konnte ich im Zytoplasma der Knorpelzellen ausser dem schwarz imprägnierten Golgiapparat, welcher als gut umschriebenes Knäuel auf einer Seite des Kerns lokalisiert ist, zahlreiche, ebenso schwarz imprägnierte Fäden, Stäbchen und Granula finden (Textfig. I-Is). Dass diese zahlreichen Gebilde die Mitochondrien der Knorpelzelle sind, wird aus dem folgenden Experiment gesichert. Die schwarz imprägnierten Bildungen der Kolatchev-Präparate wurden zunächst durch Rubaschkin'sche Methode vollkommen gebleicht; dann wurden die gebleichten Präparate nach Kull Anilinfuchsin-Aurantia gefärbt und mikroskopiert. Wir finden ohne weiteres, dass die zuerst schwarz gefärbten Fäden, Stäbchen und Granula nun mit Fuchsin rot gefärbt worden sind. Dabei wird der ebenso gebleichte Golgiapparat nun als Golgizone blass angefärbt. Ich bin in der vorliegenden Untersuchung sogar zur Ansicht gekommen, dass die Kolatchev'sche Osmiumimprägnationsmethode eine der besten Methoden für die Darstellung der Mitochondrien, mindestens der menschlichen Knorpelzellen, sein kann. Nach meiner Erfahrung ist die Unterscheidung der Mitochondrien von dem Golgiapparat nicht schwer, obwohl beide Bildungen gleich schwarz imprägniert sind. Wie eben erwähnt, werden die Golgielemente (-fäden oder -stränge) durch die Mitochondrienfärbung, z.B. Fuchsinfärbung nach Kull nicht angefärbt. Sie sind der ganzen Länge nach nicht gleichmässig 
gebaut und führen mehr oder weniger gut ąusgeprägte Verdickungen, dagegen zeigen die fadenartige Mitochondrien glatte Kontur und sind gleichmässig gebaut. Die Dicke ist bei den Golgielementen ein wenig dicker ais bei den fadenartigen Mitochondrien. Drittens stellen die Elemente des Golgiapparates, wie oben beschrieben, miteinander anastomosierend immer ein neben dem Kern liegendes, gut umschriebenes Knäuel oder Netz dar, während die Mitochondrien, wie unten erwähnt, im ganzen Zytoplasma einzeln verteilt sind, Diese eben angegebenen drei Unterschiede stimmen mit den von Deineka (1916) abgegebenen vollkommen überein.

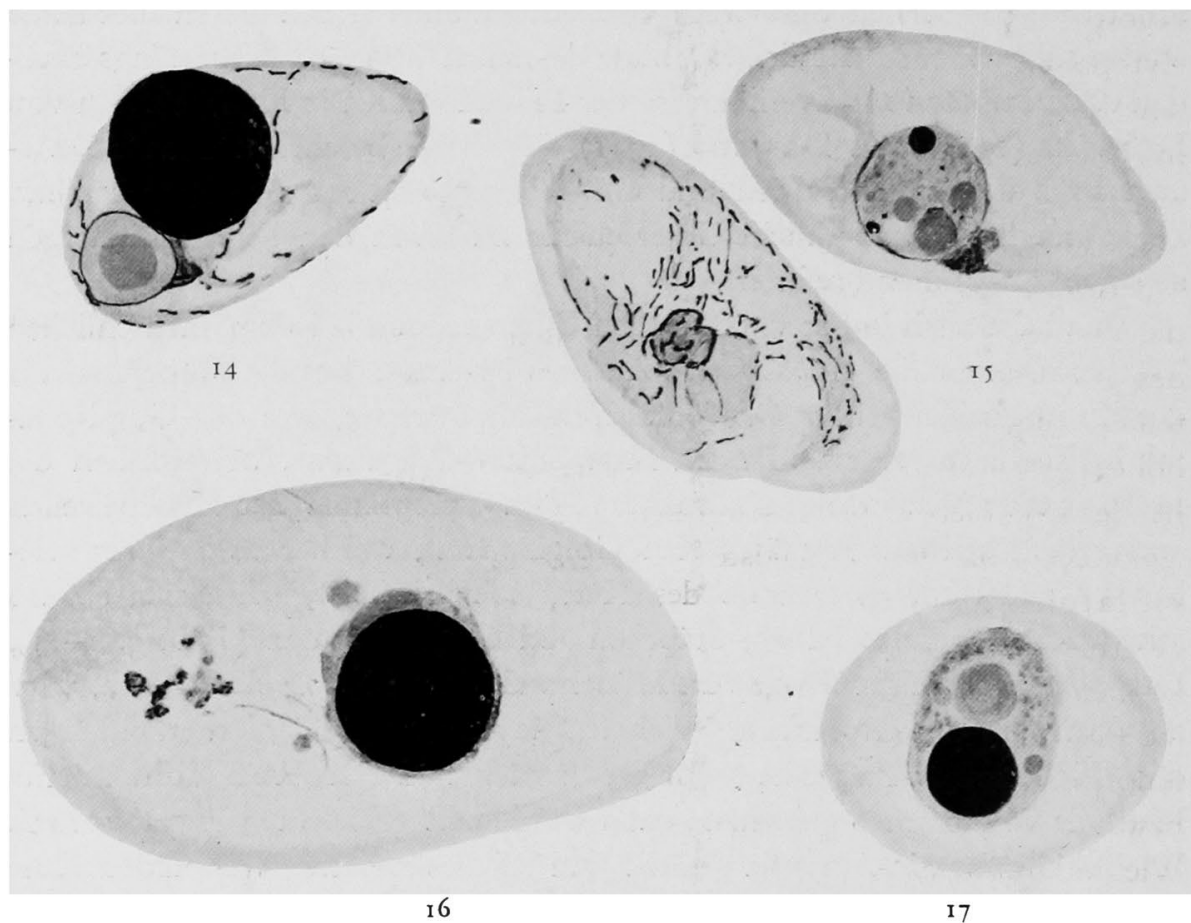

Textfig. I4-17. Knorpelzellen aus der tieferen Schicht des Trachealknorpels, Mensch. Kolatchevsche Osmiumimprägnationsmethode. Der Kern enthält grosse Nukleolen und bei is (oben), I6 und 17 Fettropfen. Der Golgiapparat ist bei I4 und Is (oben) stark rückgebildet, bei I 6 und 17 nicht mehr gefunden. Zeiss Apochr.-Imm. 2 mm, n. A. 1.3, Komp.-Ok. I 2.

Wie oben erwähnt, besteht das Chondriom der menschlichen Knorpelzellen aus Fäden, Stäbchen und Granula. Bei den in der Randzone des Trachealknorpels liegenden spindelförmigen und länglich ovalen Knorpelzellen sind die Mitochondrien nahezu gänzlich granulär (Textfig. I). Nach der Tiefe des Knorpels kommen in den nach und nach sich vergrössernden, 
rundlich werdenden Knorpelzellen in allmählich zunehmender Zahl die stäbchenförmigen Mitochondrien vor (Textfig. 2 und 3). Bei den im Zentralteil des Knorpels befindlichen grossen Knorpelzellen findet man ausser den granulären und stäbchenförmigen Mitochondrien sehr lange fadenförmige in grosser Zahl, welche mehr oder weniger langsam geschlängelt sind. Nach Daw son (1931) sind die Mitochondrien in jungen, spindelförmigen Knorpelzellen granulär, in den intermediären kurz stäbchenförmig und filamentär, in den tieferen rundlichen filamentär. Also stimmen seine Befunde mit den meinigen gut überein. Wie oben erwähnt, werden die Knorpelzellen nach der tieferen Zone zu vakuolär, so strahlen die schmalen Zytoplasmastränge von der Golgizone nach der Peripherie der Zelle zwischen den Vakuolen radiär aus (s. oben). Die Mitochondrien sind ausschliesslich in diesen Zytoplasmasträngen angeordnet, sie sind, von der Golgizone ausgehend, nach der Peripherie der Zelle radiär verteilt, wie bei den Textfig. 3-Is gesehen. Wir finden ausserdem in der Randzone der Zelle die der Oberfläche derselben parallel verlaufenden Mitochondrien. Wenn man also die Verteilung der Mitochondrien beobachtet, so versteht man das Verhälinis des Zytoplasma der Knorpelzelle sehr klar. Nach der strangartigen Verteilung des Zytoplasma ähneln die Knorpelzellen den Pflanzenzellen in hohem Masse. Zwischen den Zytoplasmasträngen finden sich hell aussehende Vakuolen, welche scheinlich die Flüssigkeit enthalten. Dass diese Vakuolen von den aufgelösten Fettropfen unabhängig sind, ist bei den Kolatchev-Präparaten gut zu ersehen, weil die Fettropfen dabei schwarz gefärbt nahezu vollkommen erhalten sind. Aus obigen Erwähnungen können wir wohl schliessen, dass die grossen rundlichen Knorpelzellen, wie die Pflanzenzellen, sehr saftreich sind. Die oberflächlichen kleinen jungen Knorpelzellen enthalten meistens keine Vakuolen, mit der Vergrösserung bzw. Alterung treten im Zytolasma nach und nach zahlreiche Vakuolen auf. Wie bekannt, ist das Knorpelgewebe gefässlos, so dass die Ernährung der Knorpelzellen von der Seite des Perichondrium besonders in der tieferen Zone verhältnismässig mangelhaft ist. Nach meiner Meinung scheint die Vakuolisierung der tieferen, älteren Knorpelzellen von diesem Verhältnis abhängig zu sein.

Wie oben erwähnt, geht der Golgiapparat bei der Degeneration der Knorpelzellen allmählich verlustig. Wir können solche Zellen auf den Textfig. I0-17 finden. Bei Betrachtung der Figuren können wir erkennen, dass die Mitochondrien im späteren Stadium der Rückbildung des Golgiapparates auch gänzlich verschwinden (Textfig. I5, I6, 17), während sie bei den Zellen in den Fig. I 2, I 3, I4 noch erhalten sind. Bei den mit sehr grossen Fettropfen, wie Fettzellen, stark gefüllten Knorpelzellen, bei welchen der 
Golgiapparat der Rückbildung anheimfällt, ist die Entwicklung der Mitochondrien auch schlecht (Textfig. 8, 9).

\section{Fett.}

Im allgemeinen enthält das Knorpelgewebe reichliches Fett; nach Leydig (1866), welcher als erster auf die Anwesenheit des Fettes in Knorpelzellen bei den Nagern hingewiesen hat, sieht das Knorpelgewebe auf den ersten Blick wie Fettgewebe aus. Seit Leydig wurde von zahlreichen Autoren das Fett in Knorpelzellen bei Menschen und verschiedenen Tieren beschrieben. Das nähere Literaturverzeichnis kann man bei Schaffer (1930) finden, so gebe ich hier nur die Arbeiten von Toldt (1877), Renaut (1893), Sacrerdotti (1907), Bergen (1904), Arnold (1908b, 'I4), Rabe (1970), Zaccarini (1910), Nagamatsu (1924), Wlassico (1930), Okuyama (1930), Dawson (1931), Murata (1933a) und Borghesse (1937) an. Nach den Ergebnissen dieser Autoren kommt das Fett in den Knorpelzellen in der Regel im Zytoplasma als Tropfen oder Granula von verschiedenen Grössen vor. Durch die eingehende Untersuchungen von Sacrerdotti, Wlassico, Okuyama u.a. wurde festgestellt, dass das in den Knorpelzellen enthaltene Fett eine normale und konstante Substanz darstellt. Nach Sacrerdotti nimmt das Knorpelfett während der Inanition nicht ab, falls die Ernährung der Knorpelzelle eine tiefe Veränderung nicht erfahren hat, während es bei der Regression der Knorpelzellen, wie von Okuyama späterhin bestätigt, abnimmt,

In der vorliegenden Untersuchung habe ich das Fett der Knorpelzellen bei den Trachealknorpeln von erwachsenen Menschen untersucht und konnte die Angabe von Sacrerdotti, Wlassico und Okuyama, dass das Fett einen normalen und konstanten Zytoplasmaeinschluss darstellt, bestätigen. Für die Untersuchung des Fettes ist die Untersuchungsmethode sehr wichtig; ich habe die mit Sudan III gefärbten Gefrierschnitte und die KolatchevPräparate beobachtet. In den letzteren weisen die Fettröpfchen oft unregelmässige eckige Form an Stelle der kugligen auf, was meiner finsicht nach ein infolge der Schrumpfung oder teilweiser Auflösung hervorgerufenes Kunstprodukt darstellt. Die Fettropfen tingieren sich bei Sudan-Färbung orangerot oder rot (Tafelfig. I-4), bei Kolatchev'scher Osmierung schwarz (Textfig. 2-I4).

Die Grösse der einzelnen Fettropfen ist nach den Zellen verschieden, sie kann selbst in einer und derselben Zelle stark varieren. In der Regel sind die Fettropfen in den kleinen spindelförmigen oder ovalen Zellen der Randzone des Knorpels klein; wir finden dort niemals grosse Fettropfen vor (Tafelfig. I, 2, Textfig. 2, 3). Nach den tieferen Schichten des Knorpels zu 
nimmt die Grösse nach und nach zu, wie von Okuyama angegeben; wir finden in den grossen rundlichen Zellen in der tieferen Zone häufig sehr grosse Fettropfen (Tafelfig. 4, Textfig. 4, 8, und 9), welche an Grösse den Kern mehrfach übertreffen. In der Textfig. 8 und 9 trifft man solche Zelle an, bei welcher der ausserordentlich grosse Fettropfen, wie bei der Fettzelle, den grössten Teil des Zelleibes einnimmt, so dass der Kern in der Peripherie stark verdrängt und abgeplattet ist. Nach Wlassico ist die Grösse der Fettropfen der Zelle des hyalinen Knorpelgewebes von dem Grade der Ernährung des Individuums abhängig. Die in der vorliegenden Untersuchung benutzten Trachealknorpel stammten alle von gesunden, gut ernährten Hingerichteten ab, somit soll die Grösse der hier in Betracht kommenden Fettropfen immer der maximalen Grösse angehören. Die Zahl der Fettropfen in einer Zelle ist im allgemeinen nicht so gross; sie sind im Zytoplasma verstreut $z u$ finden.

Aus obigen Ergebnissen können wir bezüglich des Fettgehaltes der hyalinen Knorpelzellen folgendes anführen. Die Menge der Fettropfen nimmt von den kleinen Knorpelzellen der oberflächlichen Zone nach den grösseren der tieferen, also, wie von $\mathrm{S}$ acrerd otti angegeben, mit dem physiologischen Wachstum der Zelle nach und nach zu. Unter den kleinen Zellen der oberflächlichen Zone gibt es aber oft keinen Fettropfen führende Zellen (Textfig. I). In der tieferen Zone, besonders im Zentralteil des Knorpels findet man auch fettfreie Zellen vor. Es handelt sich bei dem letzteren Fall um die in Degeneration gefallenen Knorpelzellen, bei welchen, wie oben erwähnt, der Golgiapparat vollständig oder nahezu vollständig, die Mitochondrien vollständig verschwunden sind und das Zytoplasma ganz blasig aussieht (Textfig. 15-17). Nach Sacrerdotti und Okuyama nimmt das Fett der Knorpelzellen bei ihrer Regression allmählich ab, um schliessiich gänzlich zu verschwinden. Somit konnte ich hier die Angabe der beiden Autoren bestätigen. Bei solchen regressiv veränderten Knorpelzellen habe ich in dem Kern, welcher an Grösse ausserordentlich zunimmt und grob granulär aussieht, Fettropfen gefunden, die zuweilen sehr gross werden können (Textfig. I 5-17). Das Vorkommen der Fettropfen im Kern der Knorpelzeilen ist aber von Autoren, wie Okuyama u.a., negiert. Ich aber betrachte hier es als ein Zeichen der regressiven Veränderung der Knorpelzellen.

Okuyama hat berichtet, dass bei den das Fett reichlich enthaltenden Knorpelzellen das Fett diffus oder als Granula in Knorpelkapsel, zuweilen im Zeilhof auftritt. Ich habe auch bei den grossen, das Fett in grosser Menge enthaltenden Knorpelzellen in der Peripherie des Zytoplasma oder in der Knorpelkapsel oft sehr feine, rot angefärbte Fettröpfchen zahlreich gefunden, wie in der Tafelfig. 4 gezeigt. Nach Okuyama handelt es sich bei 
diesem Fett um das von der Knorpelzelle nach der Grundsubstanz abgegebene Fett. Ich kann hier aber über die Bedeutung dieser feinen Fettröpfchen nichts Sicheres äussern. Wenn die Annahme, dass das in der Knorpelzelle enthaltene Fett als ein zur Ernährung des Knorpelgewebes bestimmter Reservenährstoff sei, zutreffend ist, so scheint die Annahme von Oku y a ma auch richtig zu sein.

Über die Bildung der Fettropfen innerhalb der Knorpelzelle haben die Autoren bis jetzt nichts näheres geäussert. Ich habe in der vorliegenden Arbeit einen wichtigen Befund getroffen. Wie schon oben beschrieben, kommen in der Nähe des Golgiapparates oder innerhalb desselben der grossen Knorpelzellen kleine färbbare kuglige Granula in wechselnder Zahl vor, welche bei der Kolatchev'schen Osmierung bräunlich angefärbt werden. Ich konnte nun bei den mit Sudan III gefärbten Gefrierschnitten wahrnehmen, dass diese Granula mit dem genannten Farbstoff orange oder gelblich orange gefärbt werden (Tafelfig. 3 u. 4). Bei solchen Gefrierschnitten wird der Golgiapparat als violett-rot angefärbte Golgizone neben dem Kern gefunden. Es ergibt sich, dass diese fraglichen Granula sich einerseits mit Eisenhämatoxylin nach Heidenhain und Aniinfuchsin, anderseits mit Sudan III anfärben. So darf ich annehmen, dass diese Granula die Vorstufe der Fettropfen sind. Die innige topographische Beziehung zwischen diesen Granula und der Golgiapparat spricht für die Annahme, dass der Golgiapparat an der Bildung der Granula, also an der Fettbildung in der Knorpelzelle beteiligt ist (s.S. 240).

\section{Glykogen und Vakuolen.}

Das Knorpelglykogen wurde von zahlreichen Autoren sehr häufig beschrieben; ich werde hier Anführung der Literatur nicht vornehmen, man kann sie in Schaffer's zusammenfassender Arbeit (1930) ersehen. Wie von Schaffer angegeben, gehört das Knorpelgewebe den glykogenreichsten Geweben an; das physiologische Vorkommen des Glykogens in Knorpelzellen ist von niemand bezweifelt. Es bleibt heute noch dahingestellt, ob dass Glykogen im Zytoplasma im geformten Zustand oder im diffusen und gelösten vorhanden ist. Nach A rnold (1908a, 'I4) ist das Glykogen in Knorpelzellen mindestens zum grössten Teil an Granula gebunden. Dagegen nach Ehrlich (1883) ist es im lebenden und unfixierten Zustand diffus im Zytoplasma gelöst. Nach seiner Meinung wird die kuglige oder schollige Gestalt erst durch Fixierung hervorgerufen. Nach Zaccarini (I9IO) stellt das Glykogen, im Gegensatz zu Fett, im lebenden Zustand keine Tropfen dar; die tropfige Gestalt ist nichts anders als ein Kunstprodukt, welches infolge der Präcipitation bei Fixierung zustande- 
kommt. Die Beziehung zwischen Vakuolen im Zytoplasma der Knorpelzellen und Glykogen wird auch auseinandergesetzt. Nach Tretjak off (1928) erscheint bei fixierten Präparaten das Zytoplasma der Knorpelzellen vakuolär, soweit das gelöste Glykogen sich innerhalb der Knorpelzellen befindet. Nach Parat (1928) kommen die Vakuolen infolge der Auflösung des Glykogens im Zytoplasma vor. Nach Schaffer (1930) gibt es in Knorpelzellen Hohlräume, welche von Glykogen erfüllt werden, obwohl die Frage, ob solche Hohlräume präformiert sind oder durch Reagensbehandlung erst bedingt werden, dahingestellt bleibt. Das Verhältnis zwischen dem Glykogen und Fett in Knorpelzellen wurde auch erörtert. Nach Renaut (1893) ist die Menge des Knorpelglykogens bei jungen Individuen gross, mit dem Vorrücken des Alters räumt das Glykogen dem. Fette den Platz ein, indem das erstere durch Zwischensubstanz in das letztere übergeht. Die Annahme Renauts wurde aber von Zaccarini negiert; nach diesem ist das Glykogen von dem Fett ganz unabhängig.

Nach meiner eigenen Beobachtung der Carminpräparate des menschlichen Trachealknorpels entha!ten die nahezu gesamten Knorpelzellen das Glykogen in verschiedener Menge. Wie allgemein anerkannt, ist das Glykogen ein Reservenährstoff, welcher in gut ernährten gesunden Individuen reichlich enthalten ist. Die von mir benutzten Trachealknorpel stammten alle von gut ernährten gesunden Hingerichteten im lebendfrischen Zustand, so soll der Glykogengehalt der Knorpelzellen in einem maximalen Wert stehen. Wir finden sogar in den spindelförmigen Zellen der oberflächlichen Zone reichliches Glykogen. In der tieferen und Zentralzone ist der Glykogengehalt der grossen rundlichen Zellen schwankend.

In den spindelförmigen Zellen erscheint das Glykogen als feine Granula, welche im ganzen Zytoplasma, die Fettvakuolen ausgenommen, nahezu gleichmässig dicht verteilt sind, falls die Vakuolen nicht vorkommen (Tafelfig. 5). Bei den grossen rundlichen Zellen ist die Gestalt der Glykogengranula nach dem Gehalt desselben etwas verschieden; bei geringem Gehalt erscheint das Glykogen fein granulär (Tafelfig. 7), aber bei grösseren zum Teil grob granulär oder grobschollig (Tafelfig. 6). Bei solchen grossen rundlichen Zellen mit vakuolärem Zytoplasma ist die Verteilung des Glykogens gewöhnlich netzartig, indem es entlang den Zytoplasmasträngen, welche Zwischenwände der Vakuolen darstellen, angeordnet ist, was beim geringerem Glykogengehalt besonders klar beobachtet werden kann (Tafelfig. 7). Bei grösseren Gehalt scheinen die Glykogengranula oder -schollen sogar die Räume der Vakuolen auszufüllen (Tafelfig. 6). Wir finden ausser dem netzartig verteilten Glykogen meistens eine schmale Schicht in der peripheren Zone der rundlichen Zellen, welche aus feinen Glykogengranula besteht. Wir 
bemerken immer neben dem Kern einen blass rötlich violett gefärbten Zytoplasmabezirk, welcher etwas scharf begrenzt und von Glykogengranula ganz frei gelassen ist (Tafelfig. 6, 7). Dieser Zytoplasmabezirk ist nichts anders als die Golgizone (Zentroplasma). Ausserdem sind die Fettvakuolen ebenso ganz frei von Glykogengranula. Die Fettvakuolen sind rundlich gestaltet, immer scharf begrenzt und ganz leer, so dass sie von den echten Vakuolen, welche unregelmässig gestaltet und unscharf begrenzt sind, leicht unterschieden werden können (Tafelfig. 5, 6, 7).

Was nun die Beziehung zwischen dem Glykogen und den Vakuolen im Zytoplasma anbelangt, so liegt dei folgende Gedanke nahe. Nach meiner Meinung ist das Glykogen in lebenden Zellen im gelösten und ungeformten Zustand diffus im Zytoplasma verteilt; infolge der Härtung mittels Fixierungsmittel erscheint es durch Präcipitation als feine Granula oder Schollen. Wenn aber bei den grösseren Zellen die mit Flüssigkeit erfüllten Vakuolen im Zytoplasma auftreten, geht das Glykogen mindestens zum Teil in die Flüssigkeit der Vakuolen aufgelöst über. Wie oben angegeben, sind die grösseren älteren Knorpelzellen saftreich; die Flüssigkeit der Vakuolen enthält somit das Glykogen in wechselnder Menge. Bei Fixierung solcher Zellen wird das Glykogen in Vakuolen präcipitiert und bei geringerem Glykogengehalt werden die gebildeten Glykogengranula entlang der Zytoplasmastränge zwischen den Vakuolen verteilt, bei grösserem treten sie ausserdem innerhalb der Vakuolen auf. Bei dem ersteren Falle sind die Glykogengranula im allgemeinen fein, bei dem letzteren sind sie grob und oft schollig. Meiner Ansicht nach sind die Vakuolen in Knorpelzellen präformiert, enthalten das Glykogen im gelösten Zustand. Sie werden von Zwischenwände des Zytoplasma abgegrenzt, welche bei dem optischen Querschnitt als die von der Golgizone (Zentroplasma) ausstrahlenden Stränge erscheinen. Wie oben erwähnt, müssen die Vakuolen von den Fettvakuolen, welche infolge Aufösung der Fettropfen zustandekommen, streng unterschieden werden. Obwohl Zaccarini (r9ro) angenommen hat, dass die Fettropfen unter Verminderung ihrer Färbbarkeit in die Vakuolen übergehen sollen, konnte ich dies nicht bestätigen.

Ausser den oben genannten Autoren haben Retzius (I914), Saguchi (1928) u.a. das Wesen der Vakuolen in Knorpelzellen erörtert. Na ga matsu (1924) hat in den Knorpelzellen die Sekretvakuolen gefunden, deren basophiler Inhalt in die Grundsubstanz abgeschieden wird. Ich konnte leider in der vorliegenden Untersuchung solche entsprechende Bildungen nicht finden. 


\section{Zusammenfassung.}

I. In der vorliegenden Untersuchung habe ich die Hyalinknorpelzellen des erwachsenen Menschen beim in lebendfrischen Zustand fixierten Trachealknorpel zytologisch eingehend studiert.

2. Im Zytoplasma der Knorpelzellen befindet sich neben dem Kern eine gut begrenzte rundliche Zytoplasmazone, welche dem Zentroplasma oder der Zentrosphäre der Autoren entspricht. Ich konnte darin aber keine Zentriolen vorfinden. Sie stimmt der Lage, Gestalt und Grösse nach mit dem Golgiapparat überein (Golgizone).

3. Die Knorpelzellen des menschlichen Trachealknorpels führen gewöhnlich einen Kern, welcher bei normalen Zellen ein verhältnismässig kleines Kernkörperchen enthält. Selten fand ich in der tieferen Zone des Knorpels unter den grossen rundlichen Knorpelzellen zweikernige vor. Bei den Knorpelzellen des menschlichen Trachealringes konnte ich weder die mitotischen noch die amitotischen Kernteilungen wahrnehmen. Nach meiner Ansicht handelt es sich wahrscheinch beim Knorpel des erwachsenen Menschen, falls sie wirklich vorkommt, ausschliesslich um die amitotische Kernteilung; die zweikernige Zelle scheint ebenso infolge dieser bedingt zu werden.

4. Bezüglich des Golgiapparates konnte ich die Befund von Deineka (1916) bei Hyalinknorpelzellen des Menschenfetus bestätigen. Der Golgiapparat der Knorpelzellen des erwachsenen Menschen stellt ein meistenfalls gut umschriebenes rundliches Knäuel oder Netz dar. Er lagert sich auf einem Pol des Kerns, gewöhnlich der Kernmembran dicht anliegend, so dass er, wie bei den Zellen der anderen Stützgewebe, eine polare Orientierung aufweist. Die Grösse des Golgiapparates überschreitet niemals die des Kerns. Die Richtung des Golgipols der Knorpelzellen ist nach den einzelnen Zellen verschieden, so dass ich kaum eine Regel annehmen konnte. Ich denke vielmehr, dass der Golgiapparat unter. Umständen um den Kern herum verschiebbar sei. Bei den zweikernigen Zellen überbrückt der lang gestreckte Golgiapparat die beiden Kerne auf einer Seite.

5. Die Mitochondrien der Zellen des menschlichen Trachealknorpels stellen Granula, Stäbchen und Fäden dar, welche im ganzen Zytoplasma vereinzelt verteilt sind. In den jungen kleinen Zellen der oberflächlichen Schicht des Knorpels sind sie meistens granulär, in den älteren grossen Zellen der tieferen Schicht aber stäbchen- oder fadenförmig. Die Mitochondrien der Knorpelzellen von erwachsenen Menschen besitzen eine starke Osmiophilie, so dass sie bei den ungefärbten Kolatchev-Präparaten, mit 
Osmiumsäure schwarz imprägniert, zusammen mit dem Golgiapparat prächtig dargestellt werden. Sie werden aber von dem letzteren leicht unterschieden.

6. Das Fett ist als ein normaler konstanter Zytoplasmaeinschluss der Knorpelzellen zu betrachten. Es sei höchstwahrscheinlich der Reservenährstoff der letzteren. Es stellt im Zytoplasma wechselnd grosse Tropfen dar. Diese sind im allgemeinen in den jungen kleinen Zellen der oberflächlichen Schicht klein und weniger zahlreich, in den älteren grossen der tieferen grob, so dass die Menge des Fettes von der Oberfläche nach der Tiefe des Knorpels allmählich zunimmt. Die Fettropfen stammen wahrscheinlich von den neben dem Golgiapparat, zuweilen darin gefundenen Granula, welche sowohl mit Anilinfuchsin nach Kull oder Eisenhämatoxylin nach Heidenhain intensiv als auch mit Sudan III nach Kawamura-Yasaki gelblich orange tingiert werden. Der Golgiapparat scheint demnach sich an der Fettbildung in der Knorpelzelle zu beteiligen.

7. Das Glykogen stellt ebenso ein normaler konstanter Zytoplasmaeinschluss dar. Bei fixierten Präparaten stellt es bei den kleinen Zellen der oberflächlichen Schicht im Zytoplasma dicht verteilte feine Granula, bei den grossen der tieferen oft gröbere Granula oder Schollen dar. Jedoch im lebenden Zustand der Zelle scheint das Glykogen nicht geformt, sondern im Zytoplasma im gelösten Zustand verteilt zu sein. Es ist zum Teil auch im flüssigen Inhalt der Vakuolen enthalten.

8. Die grösseren älteren Knorpelzellen der tieferen Schicht führen gewöhnlich ausser den Fettropfen Vakuolen, welche auch präformierte Gebilde im Zytoplasma der Knorpelzelle darstellen sollen. Bei den vakuolisierten Knorpelzellen bildet das Zytoplasma die Scheidewände zwischen den Vakuolen, welche beim optischen Querschnitt als schmale Zytoplasmastränge erscheinen. Diese strahlen von der Golgizone (Zentroplasma) nach der Peripherie radiär aus; bei solchen Zellen sind die Mitochondrien ausschliesslich in den Zytoplasmasträngen verteilt, so dass die Vakuolen ganz hell aussehen. Die Vakuolen enthalten höchstwahrscheinlich den. flüssigen Inhalt, worin das Glykogen in verschiedener Menge aufgelöst ist. Die Knorpelzellen stellen somit, wie es bei den Pflanzenzellen häufig der Fall ist, die sehr saftreichen dar.

9. Bei der Regression der Knorpelzellen in der Zentralzone des Knorpels treten zunächst im Kern grosser Nukleolus, zuweilen sogar in einiger Zahl auf. Er kann oft eine ausserordentliche Grösse erreichen und nahezu den ganzen Raum des Kerns einnehmen. Solche Nukleolen führen oft osmiophile Granula. Beim fortgeschrittenen Zustand der Regression nimmt der Kern an seiner Grösse in hohem Masse zu, sieht grob-granulär aus; er 
enthält zuweilen Fettropfen und Vakuolen. Der Golgiapparat unterliegt dabei auch einer allmählichen Rückbildung, um schliesslich vollkommen $z u$ verschwinden. Die Mitochondrien gehen ebenso schliesslich gänzlich verloren. Das Zytoplasma der der Regression anheimgefallenen Knorpelzellen wird somit zum Ende ganz hell und blasig; der Fettropfen wird im Zytoplasma von solchen Zellen nicht mehr gefunden.

Am Schluss spreche ich Herrn Prof. S. Mochizuki und Prof. T. Taniguchi für ihre freundliche Unterstützung, und Herrn Dr. T. I to für seine liebenswürdige Leitung bei Ausführung dieser Arbeit meinen herzlichsten Dank aus.

\section{Literaturverzeichnis.}

I) Arnold, J., I908 a. Supravitale Färbung der mitochondriaähnlichen Granula in den Knorpelzellen nebst Bemerkungen über die Morphologie des Knorpelglykogens. Anat. Anz. Bd. 32. S. 361 .

2) Derselbe, I $908 \mathrm{~b}$. Zur Morphologie des Knorpelglykogens und zur Struktur der Knorpelzellen. Virchows Arch. Bd. 192. S. 266.

3) Derselbe, 19i4. Über Plasmastrukturen und ihre funktionelle Bedeutung. Jena. S. 2 I9.

4) Barinetti, L., I912. L'apparato reticolare interno e la centrosfera nelle cellule di alcuni tessuti. Boll. Soc. med.-chir. Pavia. Bd. 25. S. 273. (zit. nach Schaffer (1930))

5) Bergen, Fr. v., 1904. Zur Kenntnis gewisser Strukturbilder (Netzapparat, Saftkanälchen, Trophospongien) im Protoplasma verschiedener Zellarten. Arch. mikr. Anat. Bd. 64. S. 498.

6) Bigelow, W. S., I 879 . Notiz über den Theilungsvorgang bei Knorpelzellen, sowic über den Bau des Hyalinknorpels. Arch. mikr. Anat. Bd. I6. S. 457.

7) Borghese, E., I937. I lipidi nel processo di ossificazione. Zeitschr. Zellforsch. Bd. 25. S. 622 .

8) Bütschli, O., I877. Zur Kenntnis des Theilungsprozesses der Knorpelzellen. Zeitschr. wiss. Zool. Bd. I9. (zit. nach $\mathrm{Hammer}$ )

9) Cajal, R. y., I9I4. Algunas variaciones fisiologicas y patologicas del apparato reticular de Golgi. Trab. d. labor. d. invest. biol d. 1. univ. d. Madrid. T. 12. p. I27. (zit. nach Jacobs).

10) Comes, S., i909. Sulla natura mitochondrale dello ", apparato reticolare " delle cellule cartilaginee. Boll. Acad. Gioenia Sc. nat. Catania. Ser. 2a. fasc. 6. p. 7. (zit. nach Schaffer (1930)).

II) Derselbe, 1913. Apparato reticolare o condrioma? Condriocinesi o dittocinesi ? Anat. Anz. Bd. 43. S. 422 .

I2) Dawson, A. B., I9r3. The ,zone of Golgi “ in the cartilage cells of Necturus. Anat. Rec. Vol. 48. p. 379 .

13) Deineka, D., I9I2. Der Netzapparat von Golgi in einigen Epithel- und Bindegewebszellen während der Ruhe und während der Teilung derselben. Anat. Anz. Bd. 4I. S. 289 .

14) Derselbe; i9i6. Devéloppement des cellules osseuses dans la processus enchondral. Arch. russ. d'Anat., d'Hist. et d'Embry. T. I. p. 33 I. 
15) Dubreuil, G., I9II. Le chondriome des cellules cartilagineuses chez les Mammiféres et chez l'homme. Compt. Rend. Soc. Biol. T. 69. (zit. nach Du es berg (I 9ri )).

I6) Duesberg, J., I9ro. Les chondriosomes des cellules embryonaires du Poulet et leur rôle dans la génése des myofibrilles, etc. Arch. Zellforsch. Bd. 4. S. 602 .

I7) Derselbe, I9II. Plastosomen, "Apparato reticolare interno “ und Chromidialapparat. Ergebn. Anat. u. Entw.-gesch. Bd. 20. S. 567.

I8) Ehrlich, I883. Über das Vorkommen von Glykogen im diahetischen und normalen Organismus. Zeitschr. klin. Med. Bd. I. S. 33. (zit. nach Schaffer (1930)).

I9) Fan anas, J. R., I9I2. Nota preventiva sobre el aparato reticular de Golgi en el embrion de pollo. Trab. labor. invest. biol. Univ. Madrid. T. 12. S. 247. (zit. nach Schaffer (1930)).

20) Fell, H. B., 1925. The histogenesis of cartilage and bone in the long bones of the embryonic fowl. Journ. Morph. Vol. 40. p. 417.

2r) Flemming, W., Zellsubstanz, Kern und Zellteilung. (zit. nach Meves (rgro) und Fler off (1929)).

22) Fleroff, N., 1924. Über die direkte Teilung der Knorpelzellen. (russisch) (zit. nach Fler off $(1929))$.

23) Derselbe, 1929/30. Die amitotische Teilung der Knorpelzellen und deren Beziehung zur Histogenese und Strukturfunktion des Knorpelgewebes. Anat. Anz. Bd. 68. S. 259.

24) Hammer, J. A., i 894. Uber den feineren Bau der Gelenke. II. Der Gelenkknorpel. Arch. mikr. Anat. Bd. 43. S. 8I3.

25) Hansen, 1905. Untersuchungen über die Gruppe der Bindesubstanzen. I. Der Hyalinknorpel. Anat. Heft. Bd. 27. S. 535 .

26) Hannover, A., $x 865$. On the first formation and development of cartilage. (zit. nach Fleroff(r929)).

27) Heidenhain, M., Zur Kenntnis des hyalinen Knorpels. (zit. nach Fleroff (r929)).

28) Jacobs, W., 1927. Der Golgi'sche Binnenapparat. Ergebnisse und Probleme. Ergebn. d. Biol. Bd. 2. S. 357 .

29) Kolmer, W., I915. Über einige durch R.y. Cajals Uran-Silbermethode darstellbare Strukturen und deren Bedeutung. Anat. Anz. Bd. 48. S. 506.

30) Laguesse, E, I9I. Les chondriokontes de la cellule cartilagineuse et la structure de protoplasme. Biblio. Anat. T. 2I. p. 279. (zit. nach Deus be rg (I9I I)).

31) Lams, H., I925. L'appareil filamenteux ergastoplasmique dans les cellules cartilagineuses de la trompe d'Eustache, etc. Compt. Rend. Soc. Biol. T. 93 . p. 48.

32) Leidy, J., I849. On the intimate structure and history of the articular cartilage. (zig. nach Fleroff (1929)).

33) Leydig, I866. Traité d'histologie, trad. franc. (zit nach. Sacrerdotti).

34) Loewenthal, N., x907. Zur Kenntnis der Knorpelzellen. Anat. Anz. Bd. 30. S. I9.

35) Meves, Fr., 1907. Die Chondriokonten im ihren Verhältnis zur Filarmasse Flemmings. Anat. Anz. Bd. 31. S. 501 .

36) Derselbe, i910. Úber Strukturen in den Zellen des embryonalen Stützgewebes sowie über die Entstehung der Bindegewebsfibrillen, insbesondere derjenigen der Sehne. Arch. mikr. Anat. Bd. 75. S. 149.

37) Murata, S., 1933 a. Über den Golgiapparat der Knorpelzellen. Juzenkai-Zassi. Bd. 38. S. 3535 . (japanisch).

38) Derselbe, I 933 b. Experimentelle Untersuchungen über den Golgiapparat der Knorpelzellen. Juzenkai-Zassi. Bd. 38 . (japanisch).

39) Nagamatsu, E., 1924. Morphologische Studien über die Zellen des hyalinen Knorpels. Fukuoka-Ikadaigaku-Zassi. Bd. I7. 
40) Novik off, M., 1907. Cber den Chromidialapparat in den Zellen des Subradularknorpels von Haliotis tuberculata. Anat. Anz. Bd. 34. S. 168.

4) Derselbe, 1909. Untersuchungen über Knochen- und Knorpelgewebe. Moskau. (russisch) (zit. nach Fle roff (1929)).

42) Okuyama, Y., I930. L̇ber die Fette des menschlichen Knorpelsystems. Mitt. aus d. path. Inst. d. mediz. Fakul. Niigata. Bd. i 7 . (japanisch).

43) Parat, M. et Godin, M. R., 1925. Remarques cytologiques sur la constitution de la cellule cartilagineuse ; chondriome, vacuome et appareil de Golgi. Compt. Rend. Soc. Biol. T. 93. p. 320 .

44) Parat, M., I928. Contribution à l'étude morphologique et physiologique du cytoplasme. Arch. d'Anat. micr. T. 24. p. 73.

45) Pensa, A., rgor. Demonstration von Präparaten über einen Netzapparat in den Hyalinknorpelzellen. Verh. d. deut. anat. Gesell. i9ir. S. 205.

46) Derselbe, 1913. La struttura della cellula cartilaginea. Arch. Zellforsch. Bd. II. S. 557 .

47) Rabe, F., r9ro. Experimentelle Untersuchungen über den Gehalt des Knorpels an Fett und Glykogen. Zieglers Beiträge. Bd. 48 . S. 554 .

48) Renaut, J., I 893 . Traité d'histologie pratique. (zit. nach Sacrerdotti).

49) Derselbe, rgi I. Mitochondries des cellules globuleuses du cartilage hyalin des Mammiféres. Compt. Rend. Acad. Sci. Paris. T. is2. (zit. nach Duesberg (I9I I).

50) Retterer, E., I909. Sur la structure reticulée de la cellule cartilagineuse. Compt. Rend. Soc. Biol. T. 4 6. (zit. nach Schaffer (1930)).

5i) Retzius, G., I914. Zur Kenntnis der Struktur des Protoplasmas in den lymphatischen Zellen, den Knorpelzellen und den embryonalen Bindegewebszellen. Biol. Untersuch. N.F. Bd. I8. S. 46 .

52) Sacrerdotti, G., 1900. Über das Knorpelfett. Virchows Arch. Bd. 159. S. 152.

53) Saguchi, S., 1928. Untersuchungen über die Wechselbeziehung zwischen Karyo- und Zytoplasma. 2. Das argentophile Gebilde im Kern und seine Beziehung zum Zytoplasma. Zytol. Stud. Heft. 2. Kanazawa.

54 Samssonow, N., I9ro. Über die Beziehungen der Filarmasse Flemmings zu den Fäden und Körnern Altmanns. Arch. mikr. Anat. Bd. 75. S. 635 .

55) Schaffer, J., I901. Über den feineren Bau und die Entwicklung des Knorpelgewebes und über verwandte Formen der Stützsubstanz. I. Teil. Zeitschr. wiss. Zool. (zit. nach Fle roff (1929)).

56) Derselbe, I930. Das Knorpelgewebe. Möllendorffs Hb. d. mikr. Anat. d. Mensch. Bd. II 2 . S. 210.

57) Sieveking, H., I 892 . Beiträge zur Kenntnis des Wachstums und der Regeneration des Knorpels etc. Morph. Arbeit. I892. (zit. n. Fle roff (1929)).

58) Smirnow, A. E. v., I907. Uber die Mitochondrien und den Golgischen Bildungen analoge Strukturen in einigen Zellen von Hyalinthus orientalis. Anat. Heft. Bd. 32 . S. 146.

59) Van der Stricht, O., I887. Recherches sur la cartilage hyaline. Arch. de Biol. I887. (zit. nach Fle roff $(1929)$ ).

6o) Derselbe, 1892. Contribution à l'étude de la sphére attractive. Bull. de l'acad. royale de Belgique. 1892. (zit. nach FIeroff (1929)).

6r) Studnicka, F. K., I897. Über die Histologie und die Histogenese des Knorpels der Cyclostomen. Arch. mikr. Anat. Bd. 48. S. 606.

62) Tanaka, T., 1928. Über den Golgischen Binnennetzapparat. Gunni-Dan-Zassi. Heft. I 8 r-182. (japanisch) (zit. nach Murata). 
63) Toldt, 1877. Lehrbuch der Gewebelehre. (zit. nach Sacrerd otti).

64) Torraco, L., I914. Alcune osservazione sui chondriosomi delle cellule cartilaginee nella coda del tritone regenerante. Anat. Anz. Bd. 45. S. 459.

65) Tretjakoff, D., 1927. Die Chordascheiden der Urodelen. Zeitschr, Zellforsch. Bd. 5. S. 174 .

66) Derselbe, 1928. Das Zytozentrum und der Liparosoma-(Golgi)stoff. Zeitschr. Zellforsch. Bd. 7. S. I.

67) Wlassico, T., 1930. Über den Zusammenhang der Änderung des Fettgehaltes der Zellen des hyalinen Knorpels mit dem Ernährungsgrad des Individuums. Zeitschr. mikr.anat. Forsch. Bd. 22. S. 220.

68) Zaccarini, G., r9ı. Gleichzeitige Färbung des Glykogens und des Fettes in den Rippenknorpeln. Ztb. f. allg. Path. Bd. 2I. S. 822.

\section{Erklärung der Tafelfiguren auf Tafel I.}

Alle Figuren sind wie Textfiguren mit dem A b be'schen Zeichenapparat auf Objekttischhöhe gezeichnet und mit Zeiss Apochr.-Imm. 2 mm, n. A. 1.3, Komp.-Ok. I2, in Tubuslänge $160 \mathrm{~mm}$.

Die Präparate, welche den Fig. I-4. zu Grunde liegen, sind alle mit ro\% Formalin fixierte, mit Hämatoxylin (Hansen) und Sudan III nach Kawamura-Yasaki gefärbte Gefrierschnitte des menschlichen Trachealknorpels. Kern ist blau, Fettropfen sind orangerot gefärbt.

Fig. I-2. Kleine, spindelförmige Knorpelzellen aus der Randzone des Knorpels.

Fig. 3-4. Grössere, rundliche Knorpelzellen aus der tieferen Schicht des Knorpels.

Die Präparate, welche die Fig. 5-7. lieferten, sind alle mit Formol-Alkohol fixierte, mit Best's Carmin und mit Hämatoxylin nach $\mathrm{Hansen}$ gefärbte Zelloidinschnitte des menschlichen Trachealknorpels. Kern ist blau, Glykogengranula sind rot gefärbt. Vakuolen sehen hell aus

Fig. 5. Kleine, spindelförmige Knorpelzelle aus der Randzone des Knorpels.

Fig. 6-7. Grosse rundliche Knorpelzellen aus der tieferen Schicht des Knorpels. 


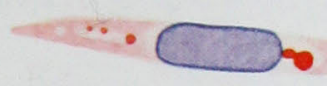

I

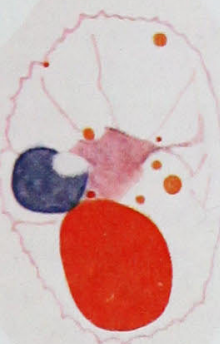

3

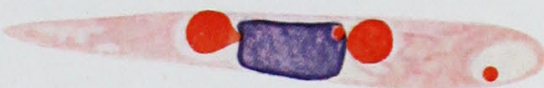

2
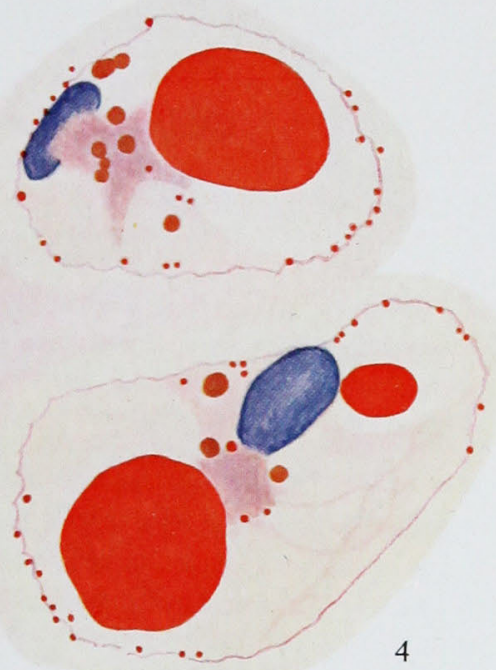

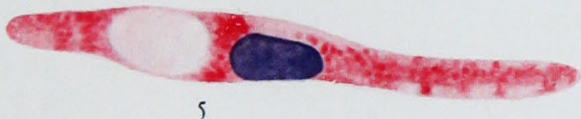

5
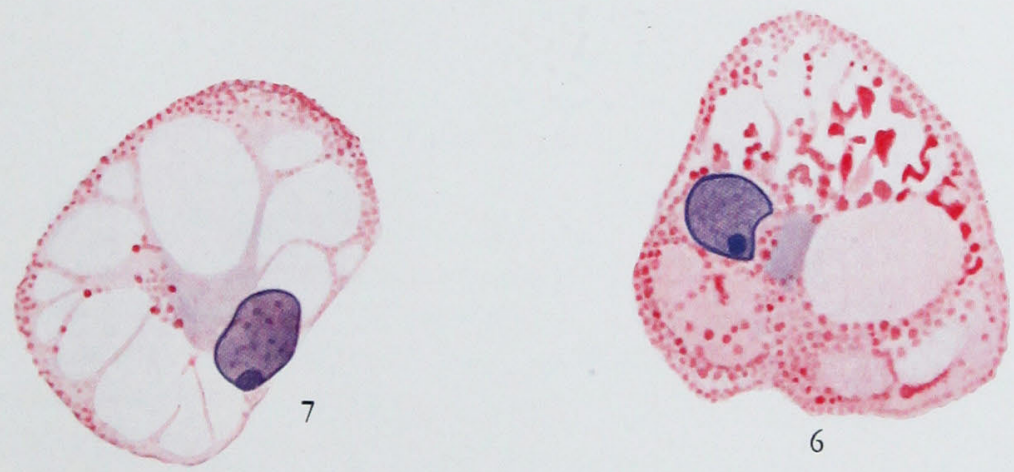

K. Oinuma. 